\title{
Alterations in Peripheral C-Reactive Protein and Inflammatory Cytokine Levels in Patients with Panic Disorder: A Systematic Review and Meta-Analysis
}

\author{
Chang-He Liu (D) \\ $\mathrm{Na} \mathrm{Hua}{ }^{2}$ \\ Huai-Yu Yang' \\ 'Department of Cardiology, Affiliated \\ Zhongshan Hospital of Dalian University, \\ Dalian, People's Republic of China; \\ ${ }^{2}$ Department of Otolaryngology, \\ Affiliated Zhongshan Hospital of Dalian \\ University, Dalian, People's Republic of \\ China
}

Correspondence: Chang-He Liu Department of Cardiology, Affiliated Zhong Shan Hospital of Dalian University, 6 Jiefang Street, Zhongshan District, Dalian, People's Republic of China

Tel +86-|347866|433

Fax +86-04II 62893555

Email liuchangheI5995I@163.com
Background: Accumulating evidence has shown the important role of the inflammatory process in the pathophysiology of mental disorders. However, the relative levels of inflammatory markers in patients with panic disorder (PD) have rarely been evaluated. The aim of the present study was to conduct a systematic review to determine the correlation of peripheral C-reactive protein (CRP) and inflammatory cytokine profiles with PD.

Methods: This study followed the Preferred Reporting Items for Systematic Reviews and Meta-Analyses guidelines. We searched for quantitative research studies published up to July 31, 2021 that measured peripheral levels of CRP and inflammatory cytokines in people with PD compared with controls. Meta-analysis using a random-effects model was performed for the levels of CRP and inflammatory cytokines with data from three or more studies.

Results: Fourteen identified studies met the inclusion criteria. In total, 18 cytokines were evaluated. Markers that were reported in more than 3 studies were included in this metaanalysis. The results showed that peripheral levels of CRP, IL-6, IL-2 and TNF- $\alpha$ were significantly higher in PD patients than in healthy controls, while there was no significant difference in peripheral levels of IL-1 $\beta$, IL-10 and IFN- $\gamma$ between groups. Notably, the relevant studies involving IL-6, IL-1 $\beta$, IL-10 and IFN- $\gamma$ in PD patients were highly heterogeneous. Similar to meta-analyses of other inflammatory factors in mental disorders, our meta-analysis also reflected differences in participant medication use, comorbid anxiety or depression, sampling methods and detection methods. Eight inflammatory cytokines were reported in only one study, and their expression levels were higher, lower, or unchanged compared with those in healthy controls.

Conclusion: There is preliminary evidence to suggest a significant inflammatory response in PD patients, but the role of inflammatory markers in PD remains unclear. Studying inflammatory markers in PD will help to clarify the etiology and pathophysiological mechanisms of the disorder.

Keywords: CRP, interleukin, TNF- $\alpha$, IFN- $\gamma$, panic disorder

\section{Plain Language Summary}

Currently, the relationship between panic disorder (PD) and peripheral inflammatory markers is unclear. It is also unclear whether the inflammatory response in PD patients is the key pathological event of PD or the stress response secondary to PD. In this study, we found that peripheral levels of CRP, IL-2, IL-6 and TNF- $\alpha$ were significantly higher in PD patients than in healthy controls, while there was no significant difference in peripheral levels of IL-1 $\beta$, IL-10 and IFN- $\gamma$ between groups. On the one hand, these findings are helpful for guiding the 
clinical diagnosis of patients with anxiety disorder; on the other hand, they can provide a theoretical basis for analyzing other pathophysiological changes caused by PD. Together, these findings have positive clinical implications.

\section{Introduction}

Accumulating evidence has demonstrated the involvement of immune-mediated inflammation in the pathophysiological process of mental diseases. ${ }^{1}$ Inflammatory cytokines are considered to be important regulators. ${ }^{2,3}$ Anxiety disorders are the most common mental disorders, ${ }^{4}$ and a high degree of comorbidity exists in various types of anxiety disorders. As a common anxiety disorder, panic disorder (PD) is a syndrome characterized by spontaneous and recurrent episodes of incapacitating anxiety and physiologic hyperarousal. It usually appears in adolescence or early adulthood, ${ }^{5}$ which seriously increases suicidal tendency, ${ }^{6}$ with negative effects on physical and occupational functions. ${ }^{4}$

PD is a very common mental disorder. The prevalence of PD varies across populations, countries and cultures, ranging from $3 \%$ to $5 \% .^{7}$ At present, $\mathrm{PD}$ is usually diagnosed with the Diagnostic and Statistical Manual of Mental Disorders questionnaires. Although research on the etiology of PD has made great progress, the underlying psychopathological mechanism remains unclear.

The inflammation hypothesis proposes that abnormal cytokines caused by the inflammatory response ${ }^{8,9}$ and immune overactivation ${ }^{10,11}$ are involved in the occurrence and development of mental disorders and are closely related to the pathophysiology of $\mathrm{PD} .^{12,13}$ It has been reported that inflammatory markers participate in the psychopathological process of PD. ${ }^{14}$ The immune process of PD patients under stress is considered an important indicator for evaluating psychopathology severity and determining the risk factors for concurrent physical diseases such as myocardial infarction. ${ }^{15}$ Abnormal peripheral inflammatory markers have been detected in PD patients, ${ }^{13}$ but their correlation with PD is currently unclear. ${ }^{16}$ As a result, the inflammatory and immune dysfunction patterns of PD need to be further confirmed. Since these inflammatory factors are related to the diagnosis of PD, the analysis of their concentration changes can be used for diagnostic indexes to reflect the degree of immune disorder and the risk factors for physical diseases in PD patients. ${ }^{15}$ Therefore, it is necessary to develop predictive objective biomarkers to assist the diagnosis of PD with subjective questionnaire reports that are commonly used in mental disorders. These biomarkers are of great significance to the diagnosis and treatment of PD and relevant physical diseases.

Although there is growing interest in the role of inflammation in mental disorders, few studies have focused on inflammatory markers in PD disease, nor have there been comprehensive analyses of their potential associations. To date, there have been two reviews on inflammatory markers in PD patients. ${ }^{9,12}$ The first one ${ }^{12}$ was a systematic review published in 2017 , which covered only a few inflammatory markers, and a meta-analysis was not performed. Another review ${ }^{9}$ did not specifically address PD, there were few relevant descriptions of PD, and a meta-analysis was not performed. These two reviews both reported changes in inflammatory markers in PD patients. To date, there has been no comprehensive systematic review and meta-analysis involving all currently published literature on PD and inflammatory markers. This study aims to systematically review the specific inflammatory biomarker profile of PD patients and conduct a meta-analysis. Our findings may provide a theoretical basis for preliminary exploration of the etiology of PD and the pathophysiology of PD-related physical diseases.

\section{Materials and Methods Search Strategy}

In this systematic review, the PubMed and Web of Science databases were searched in July 2021 (last update: 31 July 2021) for literature on the quantitative detection of peripheral blood CRP or inflammatory cytokines in PD patients and healthy controls. Then, we searched the references of the included studies to further search for studies that met the inclusion criteria. Meta-analysis was performed on some inflammatory markers. We conducted the study according to the Preferred Reporting Items for Systematic Reviews and Meta-Analyses guidelines.

The search terms used for retrieval were as follows: ("panic disorder" or "panic attacks") and (inflammat* or "C-reaction protein" or "C-reactive protein" or CRP or interleukin or IL or "tumor necrosis factor" or "tumour necrosis factor" or TNF or interferon or IFN or cytokine).

\section{Eligibility Criteria}

Cross-sectional baseline data on peripheral concentrations of inflammation markers in research participants in an unstimulated state were extracted for analyses. Longitudinal data from some longitudinal studies were excluded to avoid 
potential influences of confounding factors on the final conclusion. Studies were excluded for the following reasons: (a) the diagnosis of PD was not clear; (b) there was no definite value of peripheral inflammatory markers in PD patients or healthy controls; (c) PD patients had clinical somatic diseases; and (d) other types of studies, such as animal studies, in vitro studies, case reports, review articles and metaanalyses, were also excluded.

\section{Data Extraction and Quality Assessment}

All PD patients in these studies were assessed by standardized clinical interviews. Data extraction and quality assessment were performed on all studies that met the criteria by two independent investigators, with disagreement resolved by consensus. The following data were extracted from each included study: (a) baseline characteristics of the research participants (eg, age, sex, BMI, diagnostic methods, drug use, comorbidities) and the paper inclusion and exclusion criteria; (b) study design; and (c) measurement of biomarkers. The sample sizes of PD patients and healthy controls, and the mean value and standard deviation of each inflammatory marker between groups were extracted for meta-analysis. Study quality was evaluated using the Newcastle-Ottawa Quality Assessment Scale.

\section{Statistical Analysis}

Inflammatory markers reported in at least three studies were included in the meta-analysis. Owing to the differences in the measurement methods and expected high heterogeneity, we assessed a standardized mean difference (SMD) of each inflammation marker and performed the meta-analysis using a random-effects model, conducted using Stata 15.1 software. Each numerical value of the result is presented with a $95 \%$ confidence interval $(95 \% \mathrm{CI})$. The $\mathrm{I} 2$ statistic was calculated to quantify the heterogeneity of the included studies, with a value of $25 \%$ regarded as low, $50 \%$ as medium and $75 \%$ as high. ${ }^{17}$ The results of all analyses were considered significant if the $p$-value was less than 0.05 .

\section{Results}

\section{Search Results}

We identified 405 studies in the two databases, excluded 159 duplicate records, and screened the remaining 246 studies, of which 14 met the eligibility criteria and were included in this study. The main reasons for exclusion were animal studies, in vitro studies, and studies involving PD patients or controls combined with clinical diseases.
The process for identification and screening of the literature is shown in the systematic reviews and meta-analysis (PRISMA) flow diagram (Figure 1).

The general characteristics of the 14 eligible studies are listed in Table 1. A total of 1455 PD patients and 1385 healthy controls were involved in this study. In total, 18 different inflammatory markers were assessed (Table 2), and among them, CRP (4/14, 28.6\%), TNF- $\alpha$ (6/14, $42.9 \%)$, IL-6 (8/14, 57.1\%), IFN- $\gamma$ (4/14, 28.6\%), IL-1 $\beta$ (4/14, 28.6\%), IL-2 (4/14, 28.6\%) and IL-10 (5/14, 35.7\%) were the most commonly studied. Other cytokines were reported only in two or fewer studies.

Measurement methods were reported in all 14 studies (Table 2), including enzyme-linked immunosorbent assay (ELISA) in 7 (50\%) studies. The sensitivity of measurement was reported in 10 studies $(10 / 14,71.4 \%)$. Plasma samples were detected in 8 studies $(8 / 14,57.1 \%)$, and another 6 studies $(6 / 14,42.9 \%)$ evaluated serum samples.

\section{Risk of Bias and Quality Evaluation}

All included PD patients were diagnosed according to the Diagnostic and Statistical Manual of Mental Disorders. No significant differences in age or sex between PD patients and healthy controls were found in any of the included studies (Table 1). BMI and smoking of participants were reported in $5(5 / 14$, $35.7 \%)$ and $6(6 / 14,42.9 \%)$ studies, and no significant differences were detected (Table 1).

Comorbidity of depression and other mental disorders, and the use of psychotropic drugs are important factors that influence the inflammatory response in mental disorders. Most of the included studies (10/14, 71.4\%) excluded participants with depression or other mental disorders. Participants taking psychotropic drugs were clearly excluded in 5 studies $(5 / 14,35.7 \%)$. Three studies (3/14, $21.4 \%$ ) reported that PD patients stopped taking all antipsychotic medications within $0.5-3$ months before enrollment. Participants taking psychotropic drugs (mainly benzodiazepines) were noted in 5 studies $(5 / 14,35.7 \%)$. Only one study $(1 / 14,7.1 \%)$ did not mention the use of psychotropic drugs (Table 1).

Comorbidities of physical diseases are important determinants of inflammatory cytokines. None of the 14 studies included participants with chronic physical conditions (Table 1). Many inflammatory markers present diurnal expression patterns, and they are affected by food intake. Therefore, the sampling time and a fasting state during sampling were important factors to be considered in the 

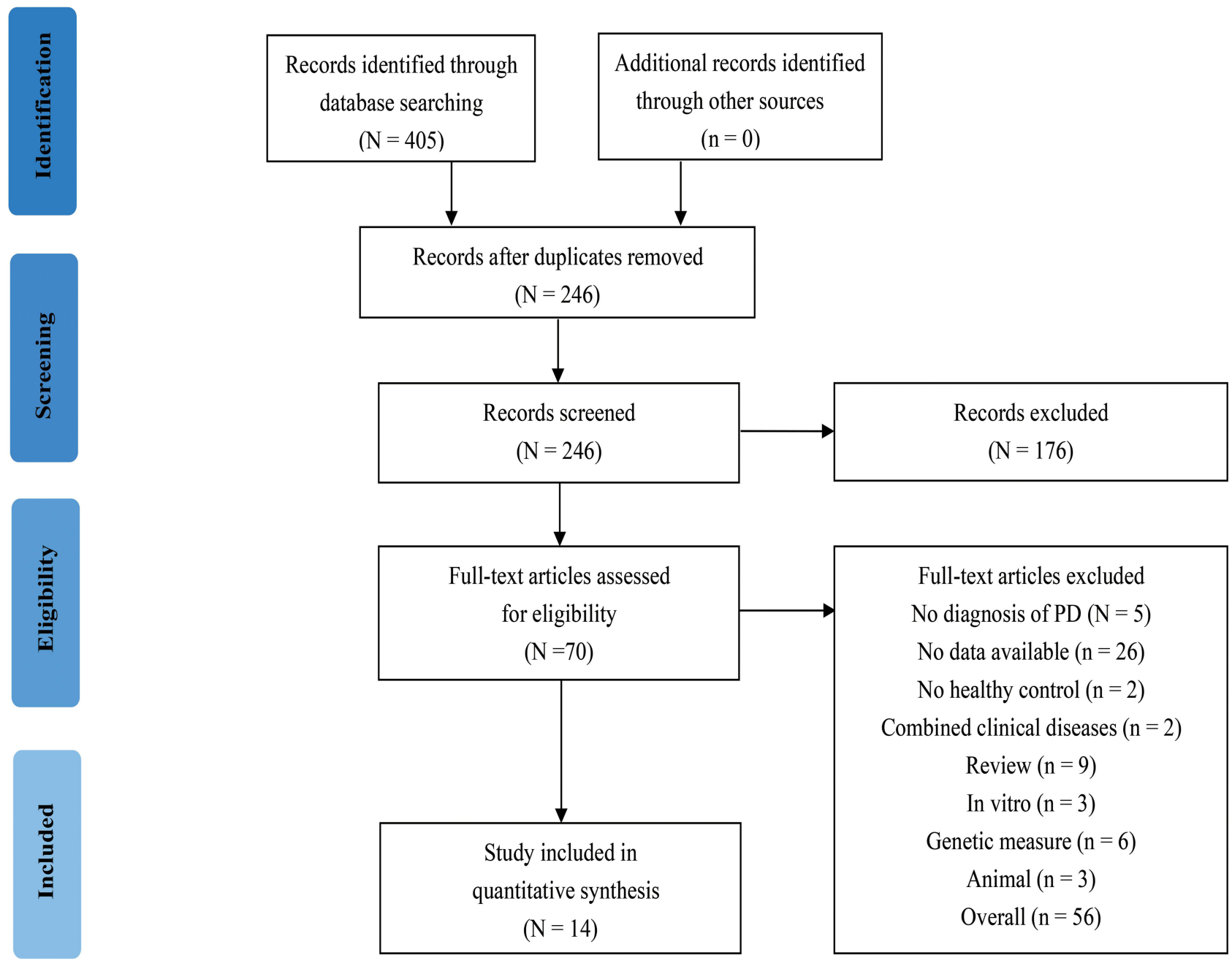

Figure I Flowchart of literature search.

analyses of cytokine levels. A sampling time of one day was recorded in 12 studies $(12 / 14,85.7 \%)$, and most of the studies $(10 / 14,71.4 \%)$ reported sampling in the morning. Fasting was clearly noted in 6 studies $(6 / 14,42.9 \%)$ (Table 2).

The overall quality of the included studies was similar, with Newcastle-Ottawa Scale scores ranging from 5 to 8 (Table 3). However, the nonresponse rate and detailed recruitment methods were not described in most studies. Considering that fewer than 10 studies were included in each metaanalysis, we did not have sufficient capacity to detect the risk of bias.

\section{CRP}

Four studies analyzed CRP in PD, involving 152 PD patients and 156 controls (Figure 2). Herrán et $\mathrm{al}^{18}$ showed that the CRP level was significantly higher in PD patients than in controls, while the remaining three studies reported by Kim et al, ${ }^{19}$
Strawn et $\mathrm{al}^{20}{ }^{20}$ and Choi et $\mathrm{al}^{21}$ did not reveal a significant difference in CRP between groups. There was little heterogeneity among the 4 studies $\left(\mathrm{I}^{2}=0.0 \%, \mathrm{df}=3, p=0.913\right)$. Our meta-analysis showed a significantly higher level of CRP in PD patients than in controls $(\mathrm{z}=3.50, p<0.001)$.

\section{Interleukins}

As shown in Table 3, a total of 11 studies illustrated the correlation between PD and interleukins (ILs).

IL-6 was the most frequently measured interleukin in the included studies, which was reported in 8 studies involving $341 \mathrm{PD}$ patients and 320 controls. The conclusion that PD patients had a significantly higher level of IL6 than controls was obtained in 4 studies, ${ }^{13,19,21,22}$ while the other 4 studies $^{15,23-25}$ did not identify a significant difference in IL-6 levels. Our meta-analysis showed a significantly higher level of IL-6 in PD patients than in controls $(\mathrm{z}=2.32, p=0.020)$, although the heterogeneity 


\begin{tabular}{|c|c|c|c|c|c|c|}
\hline 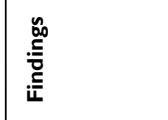 & $\begin{array}{l}\stackrel{u}{T} \\
\hat{a} \\
\underline{a}\end{array}$ & 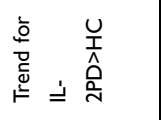 & 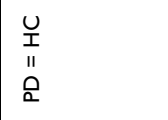 & $\begin{array}{l}\underline{u} \\
\hat{a}\end{array}$ & $\begin{array}{l}\underline{u} \\
\hat{a}\end{array}$ & 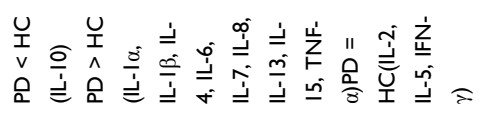 \\
\hline 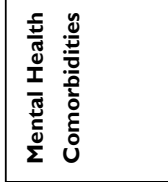 & 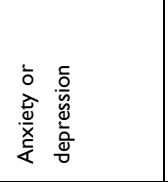 & 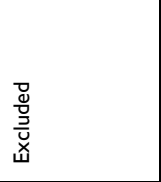 & 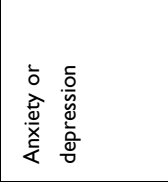 & $\frac{o x}{z}$ & 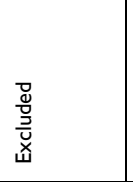 & 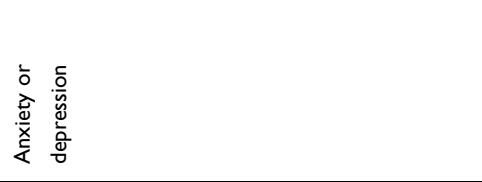 \\
\hline 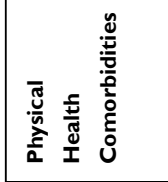 & 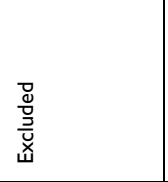 & 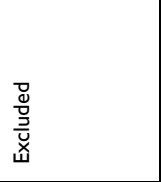 & 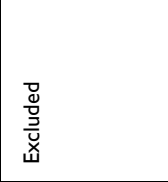 & 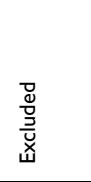 & 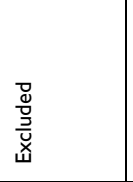 & 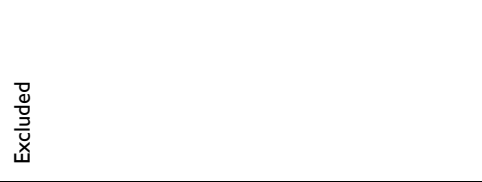 \\
\hline 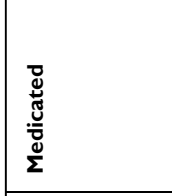 & 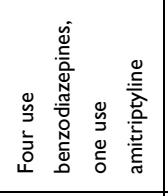 & 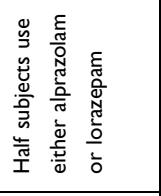 & 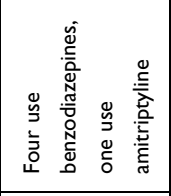 & 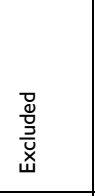 & 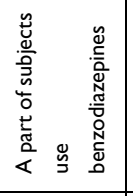 & 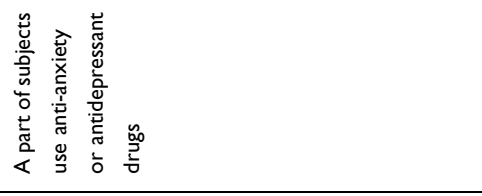 \\
\hline 䓂高 & $\frac{\infty}{z}$ & $\frac{\infty}{z}$ & $\frac{\infty}{z}$ & $\frac{\infty}{z}$ & $\frac{\infty}{z}$ & $\stackrel{o}{z}$ \\
\hline $\bar{\Sigma}_{\infty}$ & $\stackrel{o ̛}{z}$ & $\frac{\alpha}{z}$ & $\frac{\mathscr{o}}{z}$ & $\frac{\alpha}{z}$ & $\stackrel{o}{Z}$ & $\frac{o}{z}$ \\
\hline 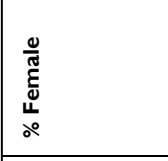 & 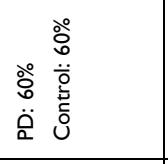 & 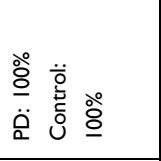 & 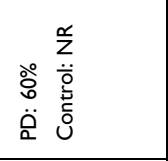 & $\frac{\infty}{z}$ & $\frac{o}{z}$ & $\frac{\alpha}{z}$ \\
\hline 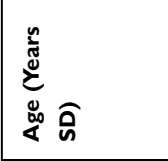 & 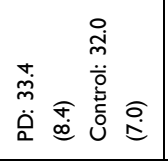 & 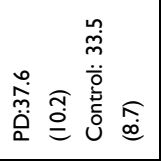 & 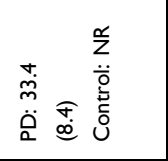 & $\stackrel{\alpha}{z}$ & $\frac{\infty}{z}$ & $\frac{o}{z}$ \\
\hline 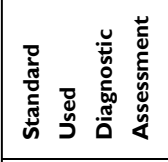 & 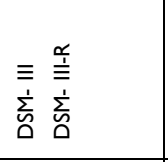 & 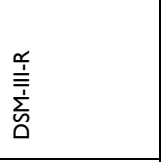 & 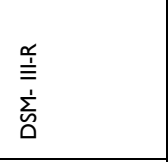 & 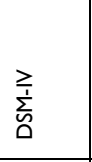 & 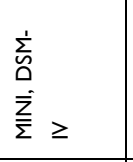 & 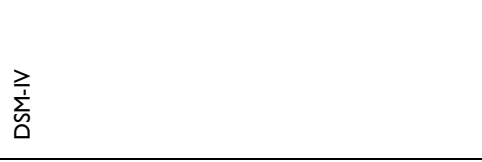 \\
\hline 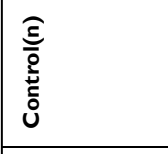 & $\circ$ & $\underline{\infty}$ & 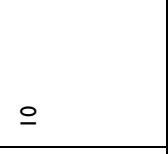 & $\circ$ & $\bar{\sim}$ & i \\
\hline$\widehat{\varrho}$ & 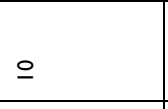 & \pm & 음 & in & m & i \\
\hline 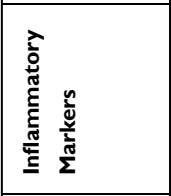 & $\stackrel{\cong}{\stackrel{一}{=}}$ & 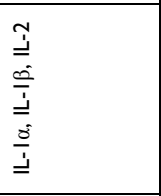 & $\stackrel{\stackrel{i}{i}}{\not}$ & $\stackrel{\infty}{\stackrel{\infty}{=}}$ & 总 & 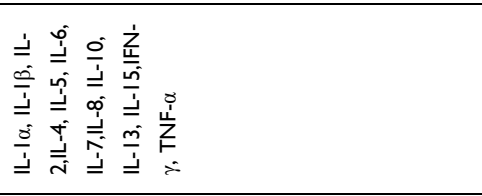 \\
\hline 密 & 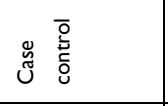 & 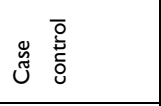 & 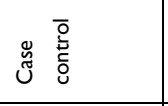 & 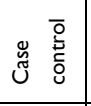 & 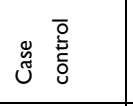 & 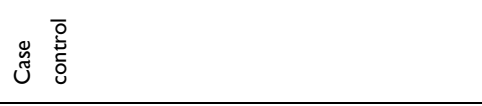 \\
\hline نे & 츨 & 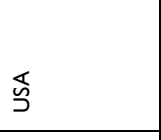 & ब्र & 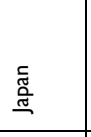 & $\begin{array}{l}\text { 离 } \\
\text { 心 }\end{array}$ & 艿 \\
\hline ते & 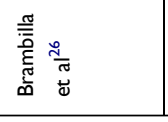 & 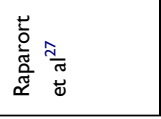 & 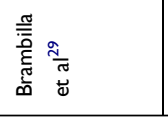 & 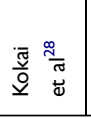 & 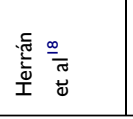 & 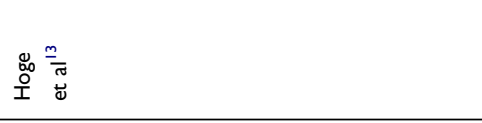 \\
\hline
\end{tabular}




\begin{tabular}{|c|c|c|c|c|c|c|c|}
\hline 离 & 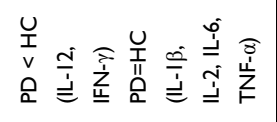 & $\begin{array}{l}u \\
\underline{x} \\
11 \\
a \\
a\end{array}$ & 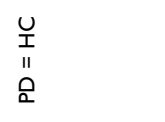 & 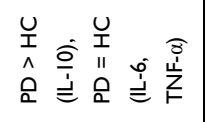 & 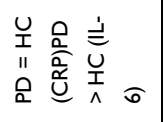 & 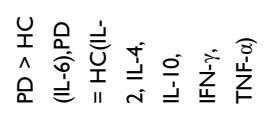 & $\begin{array}{l}\frac{u}{1} \\
\frac{1}{a} \\
a\end{array}$ \\
\hline 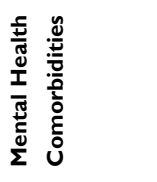 & 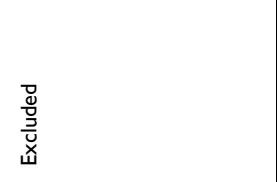 & 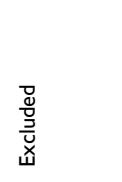 & 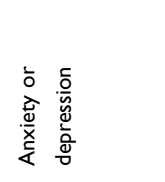 & 总 & 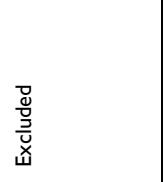 & 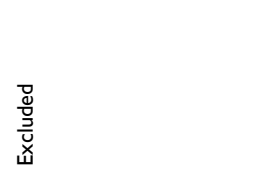 & 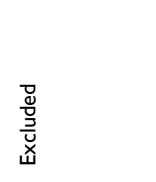 \\
\hline 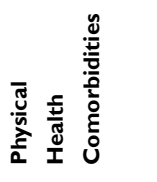 & 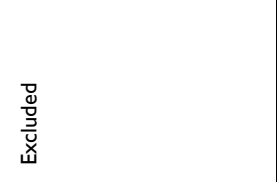 & 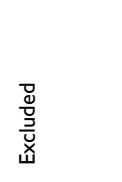 & 总 & 总 & 总 & 总 & 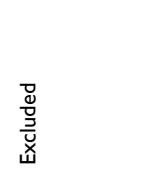 \\
\hline 逆 & $\begin{array}{l}\text { 递 } \\
\frac{\mathrm{y}}{\mathrm{x}} \\
\end{array}$ & 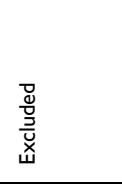 & 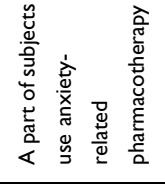 & 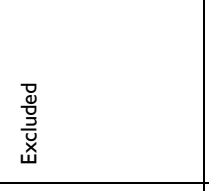 & $\begin{array}{l}\text { 总 } \\
\frac{\underline{y}}{x} \\
\end{array}$ & 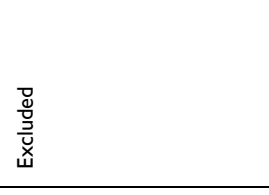 & $\begin{array}{l}\text { 总 } \\
\frac{y_{x}}{4} \\
\end{array}$ \\
\hline 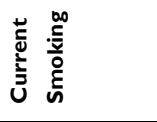 & 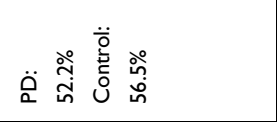 & 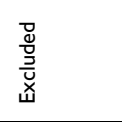 & $\frac{\alpha}{z}$ & 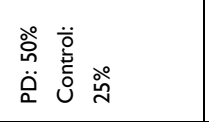 & 范总 & 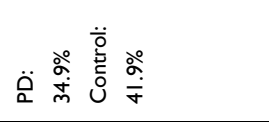 & 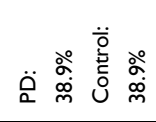 \\
\hline$\overline{\Sigma_{\boldsymbol{\omega}}}$ & $\frac{x}{z}$ & $\frac{x}{z}$ & 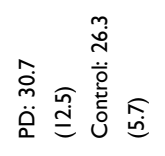 & 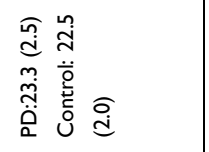 & 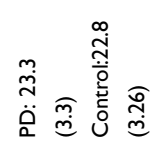 & 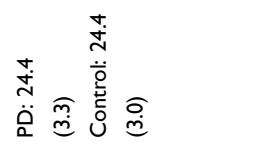 & $\frac{x}{z}$ \\
\hline 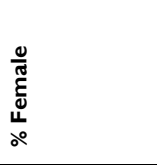 & 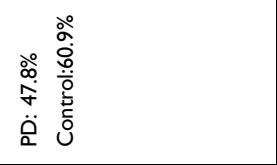 & 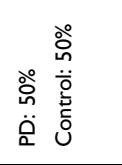 & 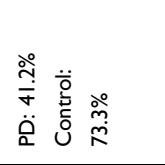 & 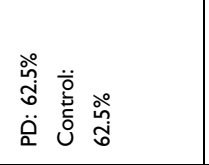 & 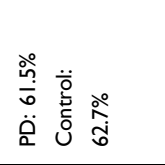 & 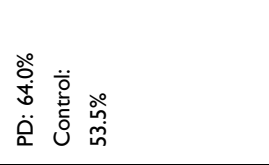 & 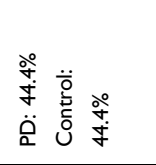 \\
\hline 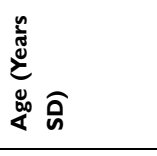 & 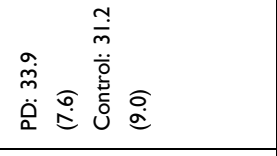 & 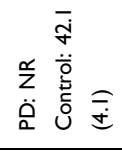 & 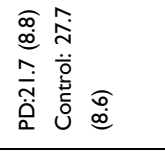 & 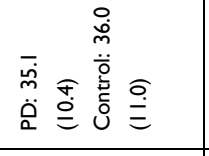 & 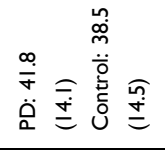 & 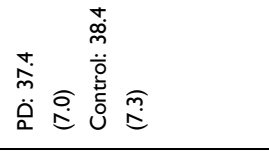 & 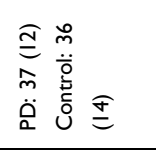 \\
\hline 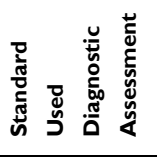 & 咅 & 咅竞 & 竞 & 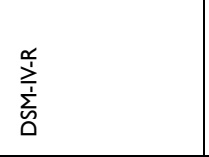 & 咅 & 胥 & 咅 \\
\hline 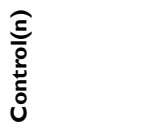 & $\approx$ & ה & $=$ & $\tilde{m}$ & in & $\infty$ & $\stackrel{\infty}{\underline{0}}$ \\
\hline छัฒ & $\approx$ & 8 & $\underline{\underline{n}}$ & $\tilde{\mu}$ & $\tilde{n}$ & $\stackrel{\infty}{\infty}$ & $\underline{\infty}$ \\
\hline 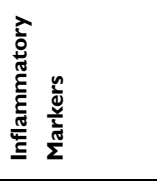 & 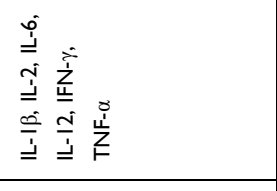 & 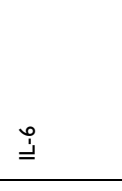 & Ũ & 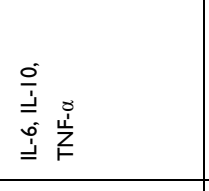 & 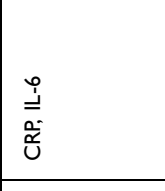 & 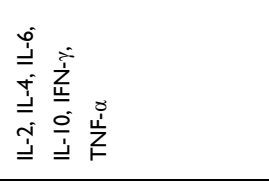 & 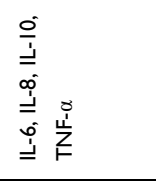 \\
\hline 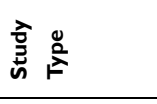 & 总 & 总 & 递毫 & 递 & 递 & 总 & 送 \\
\hline Uે & 离 & 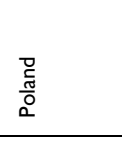 & 离 & 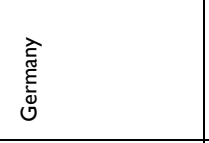 & 竒 & 胥 & 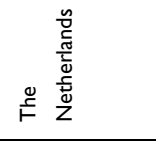 \\
\hline 錞 & 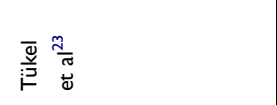 & 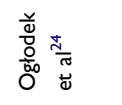 & 点递 & 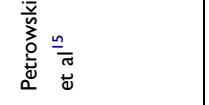 & 焉 & 곳 & 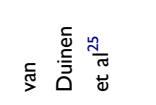 \\
\hline
\end{tabular}



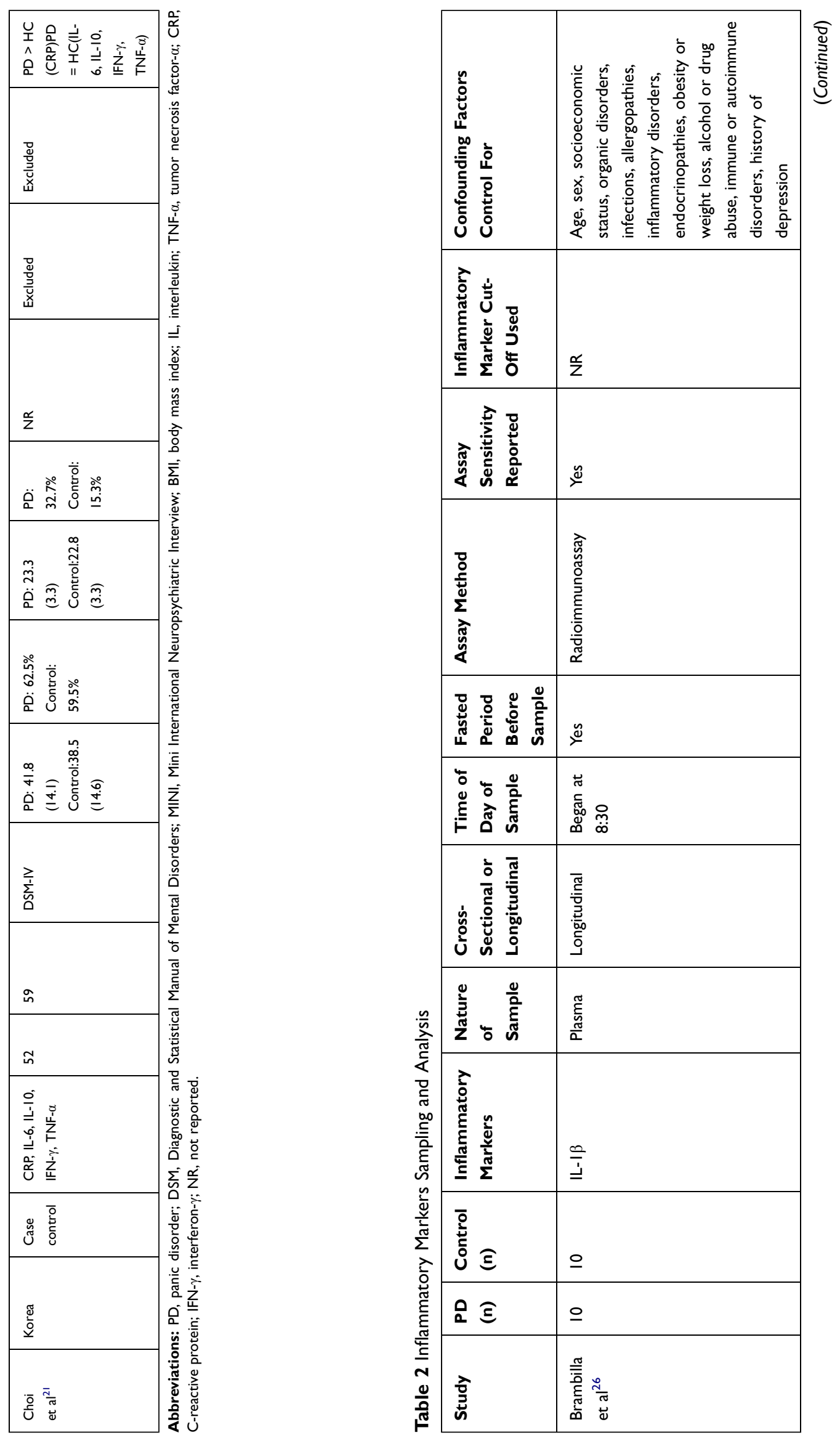


\begin{tabular}{|c|c|c|c|c|c|}
\hline 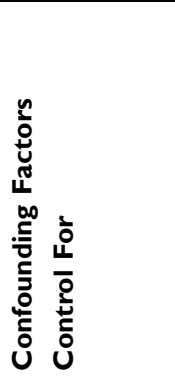 & 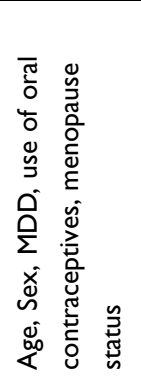 & 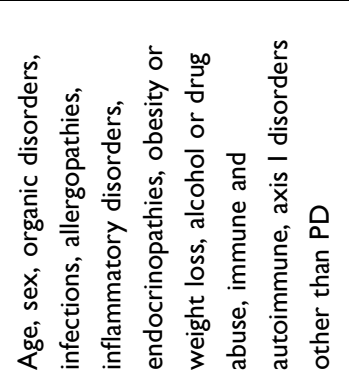 & 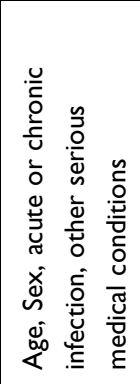 & 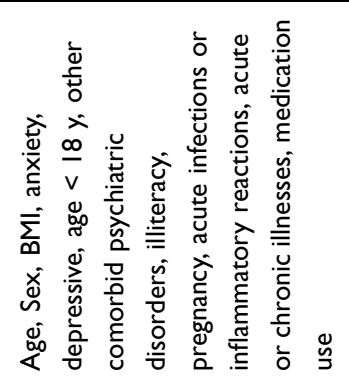 & 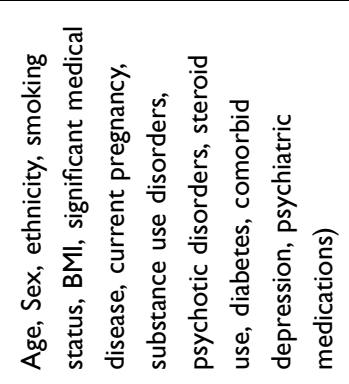 \\
\hline 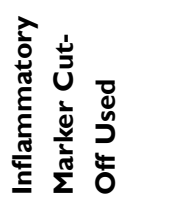 & $\stackrel{\alpha}{Z}$ & $\stackrel{\alpha}{Z}$ & $\stackrel{\alpha}{z}$ & $\stackrel{\alpha}{z}$ & 产 \\
\hline 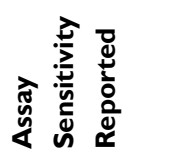 & $\stackrel{y}{\nu}$ & $\stackrel{\check{\Perp}}{\check{\nu}}$ & $\frac{\alpha}{z}$ & $\stackrel{\check{\Delta}}{\check{\nu}}$ & $\stackrel{\check{g}}{\check{\nu}}$ \\
\hline 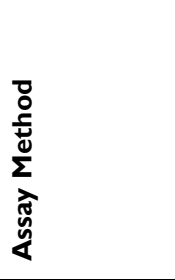 & $\stackrel{\overleftarrow{n}}{\underline{u}}$ & 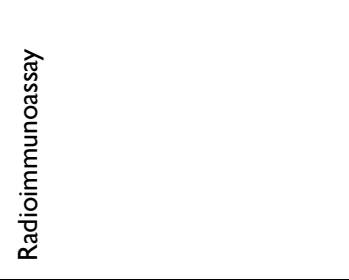 & $\frac{\overleftarrow{y}}{\tilde{u}}$ & 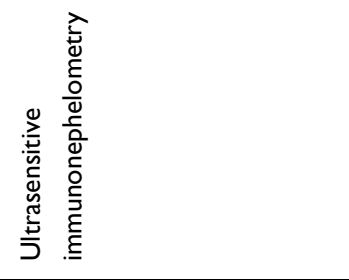 & 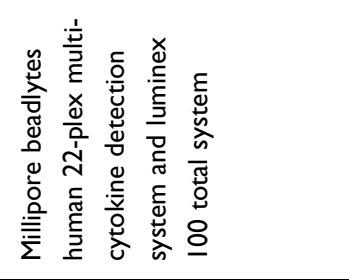 \\
\hline 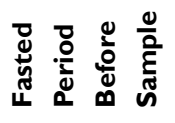 & $\frac{\alpha}{z}$ & 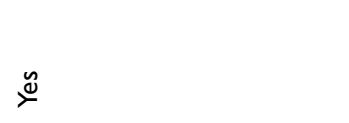 & $\stackrel{\mathscr{\alpha}}{Z}$ & 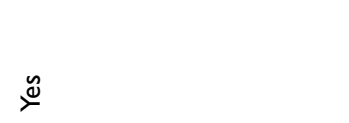 & $\stackrel{\mathscr{\alpha}}{Z}$ \\
\hline 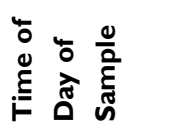 & 잉 & 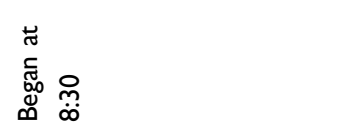 & 苍 & 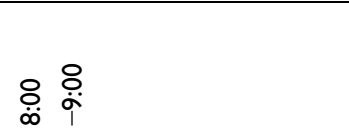 & 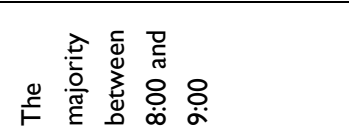 \\
\hline 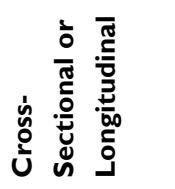 & 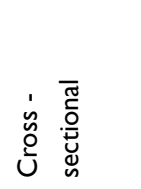 & 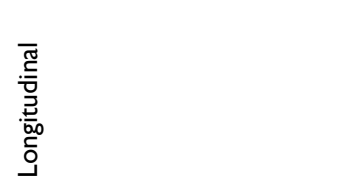 & 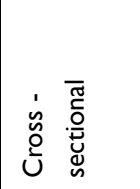 & 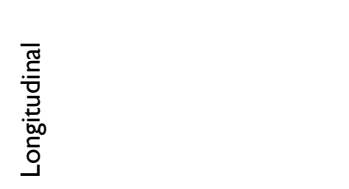 & 离 \\
\hline 竞 & $\underset{\xi}{\xi}$ & $\begin{array}{l}\frac{\pi}{\tilde{E}} \\
\frac{\pi}{\alpha} \\
\frac{\pi}{2}\end{array}$ & 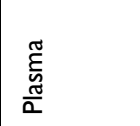 & 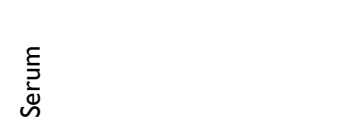 & 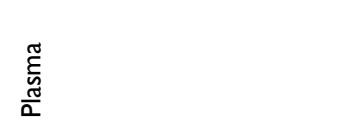 \\
\hline 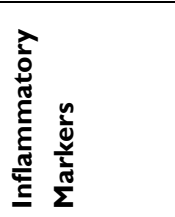 & 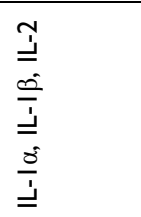 & $\underset{i}{\stackrel{i}{L}}$ & $\stackrel{\infty}{\stackrel{\infty}{=}}$ & 嵌 & 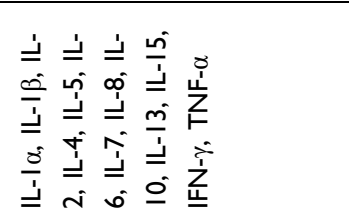 \\
\hline 㝘 & $\underline{\infty}$ & 으 & 。 & $\bar{\sim}$ & ㅇ. \\
\hline 으 & $\dot{\Psi}$ & 은 & n & $\tilde{m}$ & ㅇ \\
\hline ते & 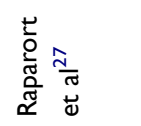 & 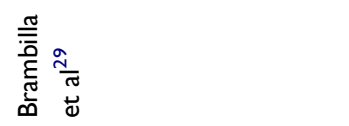 & 离 & 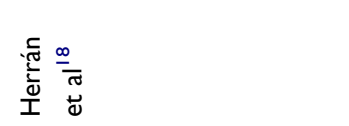 & 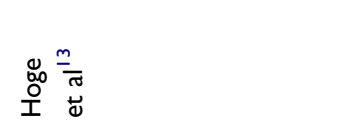 \\
\hline
\end{tabular}




\begin{tabular}{|c|c|c|c|}
\hline 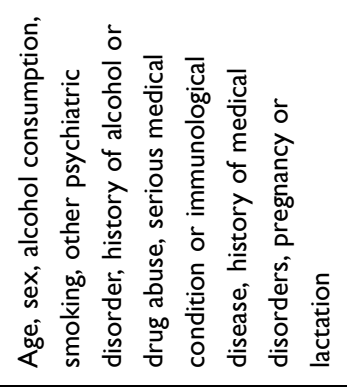 & 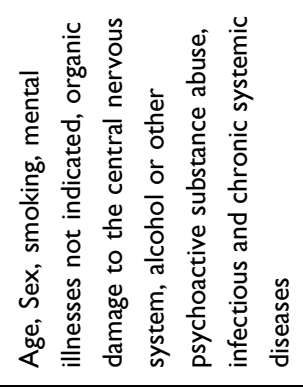 & 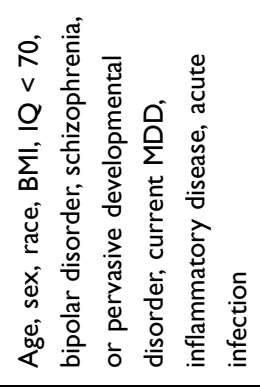 & 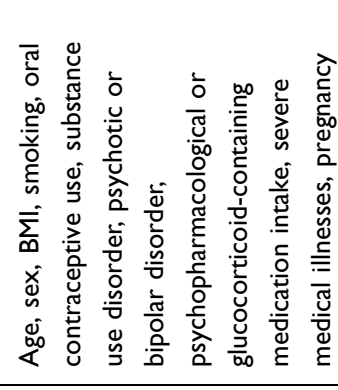 \\
\hline$\frac{\mathscr{c}}{Z}$ & $\frac{\alpha}{z}$ & $\frac{\alpha}{z}$ & $\frac{\alpha}{z}$ \\
\hline$\underbrace{\tilde{u}}$ & $\stackrel{\mathscr{g}}{\check{\nu}}$ & $\frac{\alpha}{z}$ & $\frac{\alpha}{z}$ \\
\hline 窎 & 离 & 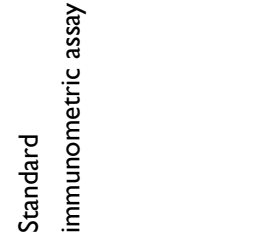 & 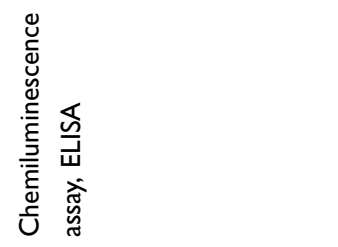 \\
\hline$\frac{\tilde{c}}{z}$ & $\stackrel{\square}{\widetilde{u}}$ & $\stackrel{0}{z}$ & $\frac{o}{z}$ \\
\hline రి & 㲾 & 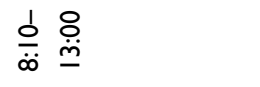 & $\frac{o}{z}$ \\
\hline 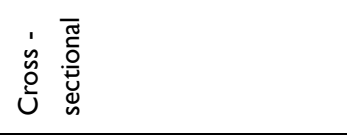 & 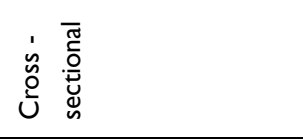 & 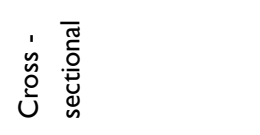 & 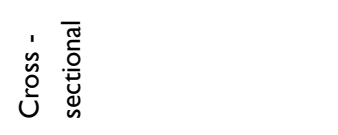 \\
\hline $\begin{array}{l}\frac{\tilde{m}}{\tilde{m}} \\
\frac{\pi}{\alpha}\end{array}$ & $\begin{array}{l}\frac{\tilde{w}}{\overline{\underline{m}}} \\
\frac{1}{a}\end{array}$ & 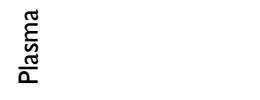 & $\begin{array}{l}\frac{\tilde{5}}{5} \\
\frac{\mathrm{m}}{0}\end{array}$ \\
\hline 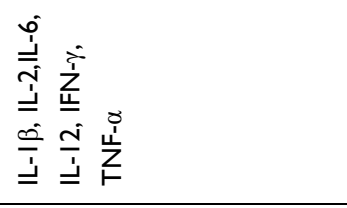 & $\stackrel{\text { İ }}{=}$ & 造 & 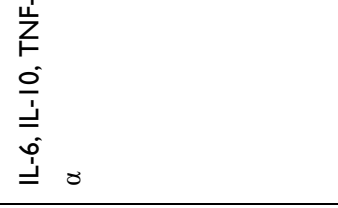 \\
\hline$\widetilde{\sim}$ & q & $\simeq$ & $\tilde{m}$ \\
\hline ఇ & 8 & $\underline{\underline{n}}$ & $\stackrel{\sim}{m}$ \\
\hline 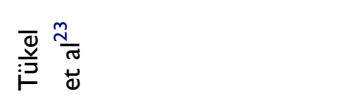 & 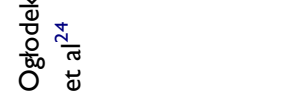 & 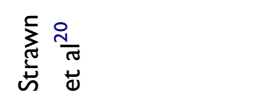 & 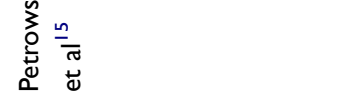 \\
\hline
\end{tabular}




\begin{tabular}{|c|c|c|c|c|}
\hline 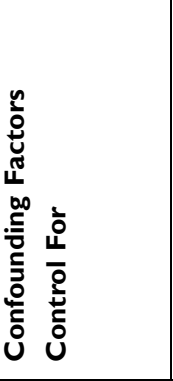 & 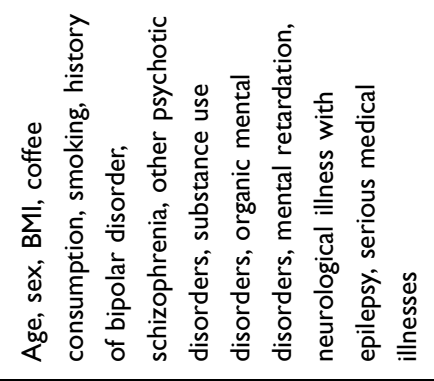 & 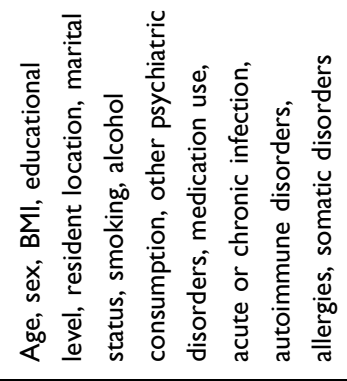 & 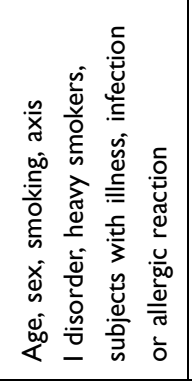 & 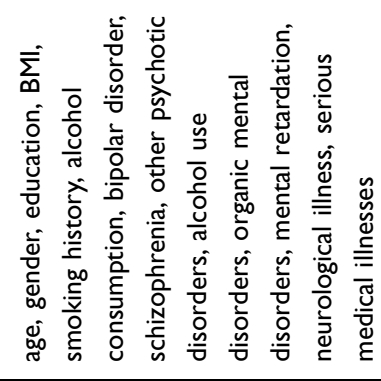 \\
\hline 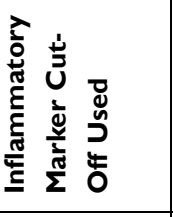 & $\frac{\alpha}{z}$ & $\frac{o}{z}$ & $\stackrel{\alpha}{z}$ & $\stackrel{\alpha}{z}$ \\
\hline 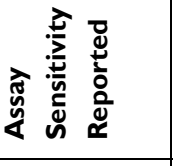 & 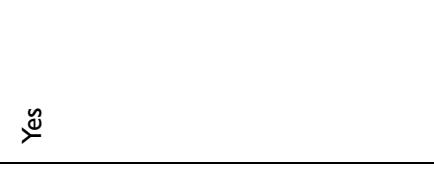 & $\frac{\alpha}{z}$ & 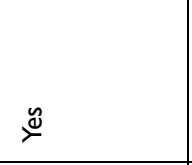 & $\stackrel{\mathscr{g}}{=}$ \\
\hline 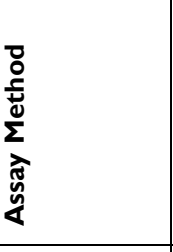 & 志 & 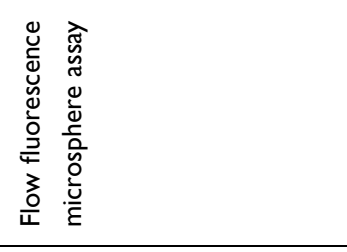 & 吾 & 崖 \\
\hline 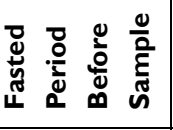 & $\stackrel{\alpha}{z}$ & $\check{\check{\nu}}$ & $\frac{\alpha}{z}$ & $\stackrel{0}{0}$ \\
\hline 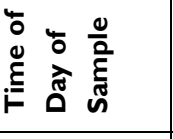 & $\frac{\alpha}{z}$ & 实芯 & 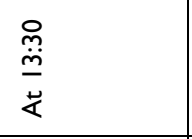 & $\stackrel{\circ}{\circ} \stackrel{\circ}{\circ}$ \\
\hline 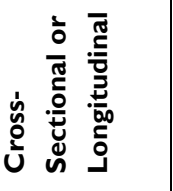 & 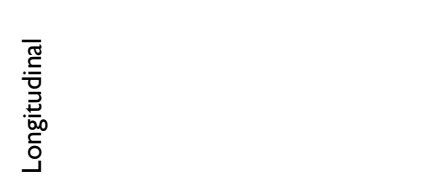 & 它 & 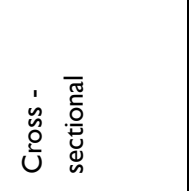 & 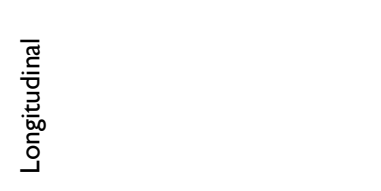 \\
\hline 总 & 衰 & 言 & 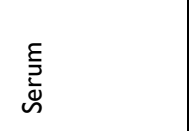 & 竭 \\
\hline 童 & 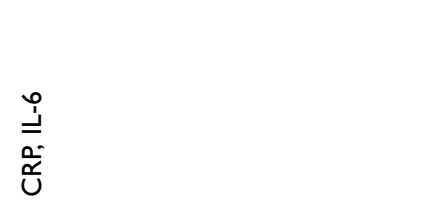 & 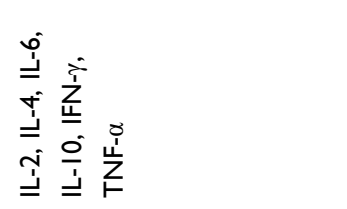 & 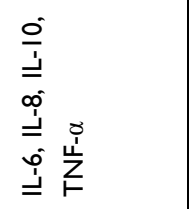 & 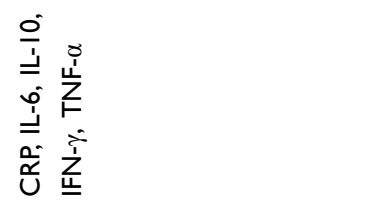 \\
\hline 离 & in & $\infty$ & $\underline{\infty}$ & in \\
\hline 오 & n & $\infty$ & $\underline{\infty}$ & in \\
\hline ) & 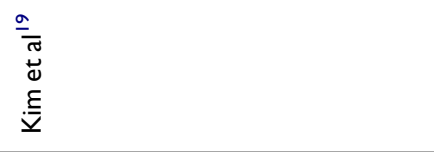 & 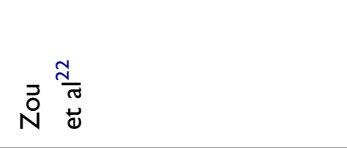 & 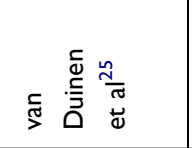 & $\overline{\bar{o}} \overline{\frac{\sigma}{\sigma}} \frac{\bar{c}}{\bar{\sigma}}$ \\
\hline
\end{tabular}




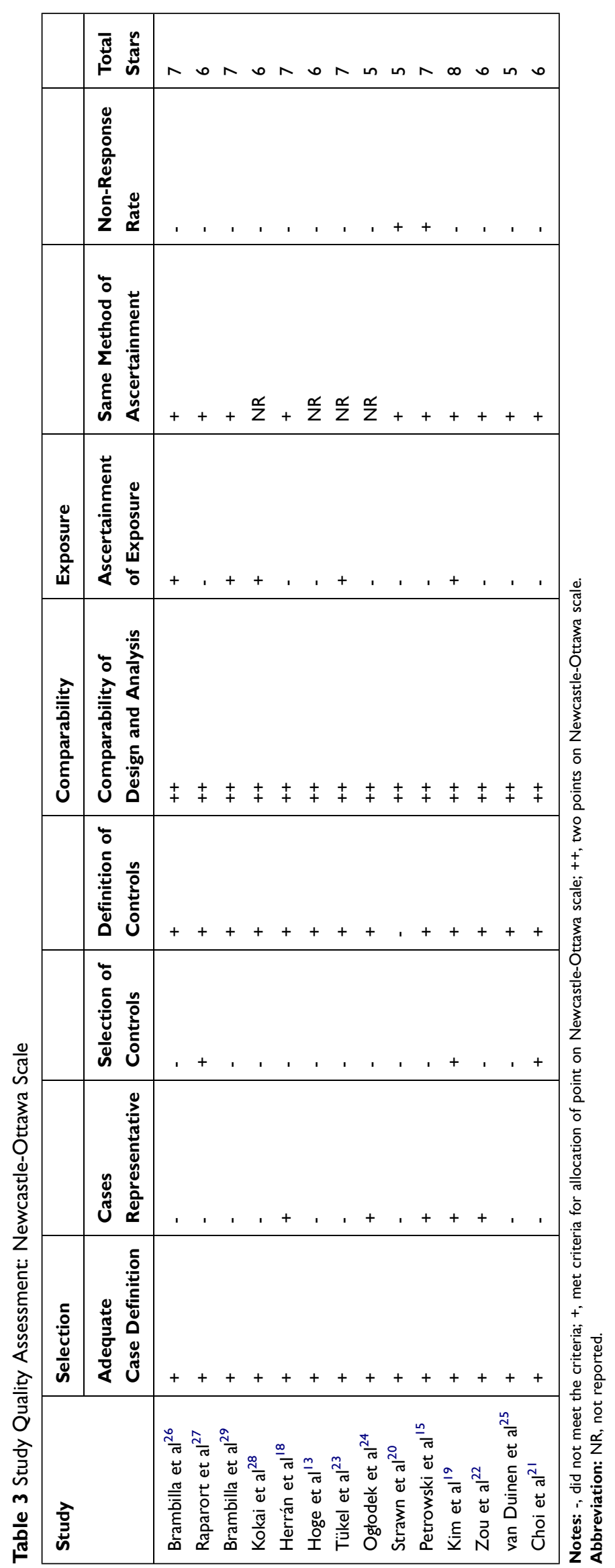




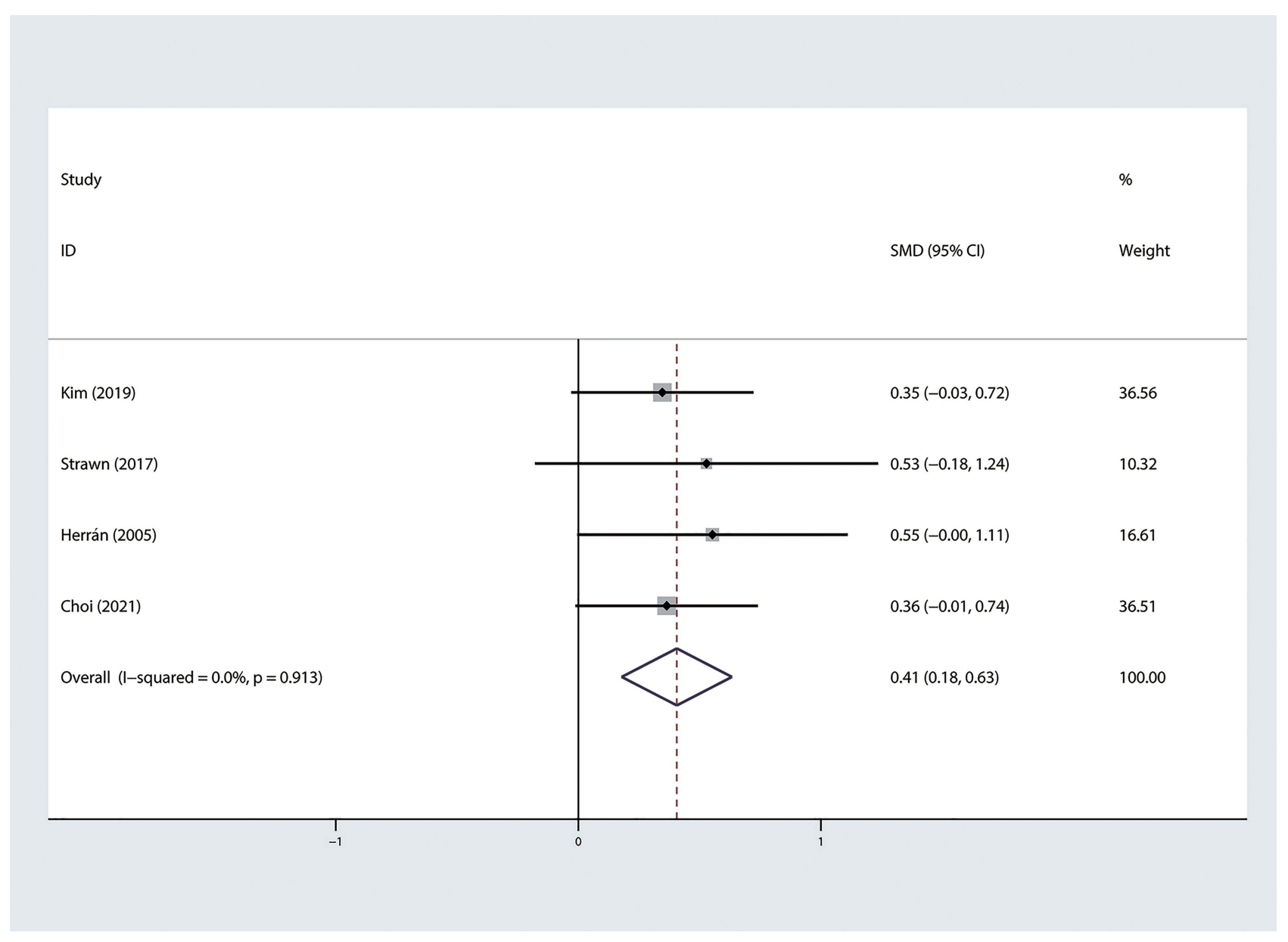

Figure 2 Forest plot of standard mean difference (SMD) of CRP levels in PD vs controls.

Abbreviations: CRP, C-reactive protein; PD, panic disorder.

was relatively high among 8 studies $\left(\mathrm{I}^{2}=73.9 \%\right.$, df $=7$, $p<0.001$ ) (Figure 3).

IL-1 $\beta$ levels in PD patients and controls were detected in 4 studies, ${ }^{13,23,26,27}$ involving 63 PD patients and 55 controls. We did not detect a significant difference in IL-1 $\beta$ levels between PD patients and controls $(\mathrm{z}=1.12, p=0.261)$, and the heterogeneity was relatively high $\left(\mathrm{I}^{2}=80.0 \%\right.$, df $\left.=3, p=0.002\right)$ (Figure 4).

Four studies compared IL-2 levels in PD patients with controls, involving $140 \mathrm{PD}$ patients and 134 controls. Raparort et $\mathrm{al}^{27}$ reported that IL-2 levels in PD patients tended to increase compared with those in the control group $(P<0.07)$. Nevertheless, no significant difference in IL-2 levels between PD patients and controls was observed in studies conducted by Tükel et al, ${ }^{23}$ Hoge et al, ${ }^{13}$ and Zou et al. ${ }^{22}$ Our meta-analysis found a higher level of IL-2 in PD patients $(\mathrm{z}=2.25, \mathrm{p}=0.025)$ (Figure 5).

IL-10 levels in PD patients were reported in 5 studies, with inconsistent findings. A total of 205 PD patients and 198 controls were involved. Zou et $\mathrm{al}^{22}{ }^{22}$ van Duinen et $\mathrm{al},{ }^{25}$ and Choi et $\mathrm{al}^{21}$ demonstrated that there was no significant difference in IL-10 levels between PD patients and controls. Inconsistently, Petrowski et al ${ }^{15}$ reported a higher level of IL10 in PD patients than in controls, while the opposite result was obtained in another study ${ }^{13}$ with the smallest sample size. Our meta-analysis suggested that there was no significant difference in IL-10 levels between groups ( $\mathrm{z}=1.40, p=0.161)$, and the heterogeneity among the 5 studies was relatively high $\left(\mathrm{I}^{2}=94.2 \%, \mathrm{df}=4, p<0.001\right)$ (Figure 6).

The correlation between PD and other ILs has rarely been reported. Only two studies compared IL-1a levels between PD patients and controls, and they obtained inconsistent results. Hoge et $\mathrm{al}^{13}$ showed a higher level of IL-1a in PD patients, while Raparort et $\mathrm{al}^{27}$ did not identify a significant difference. The IL-8 level in PD patients was also reported in 2 studies, and Hoge et $\mathrm{al}^{13}$ showed a higher level of IL-8 in PD patients than in controls, but van Duinen et $\mathrm{al}^{25}$ insisted no significant difference. Two studies 


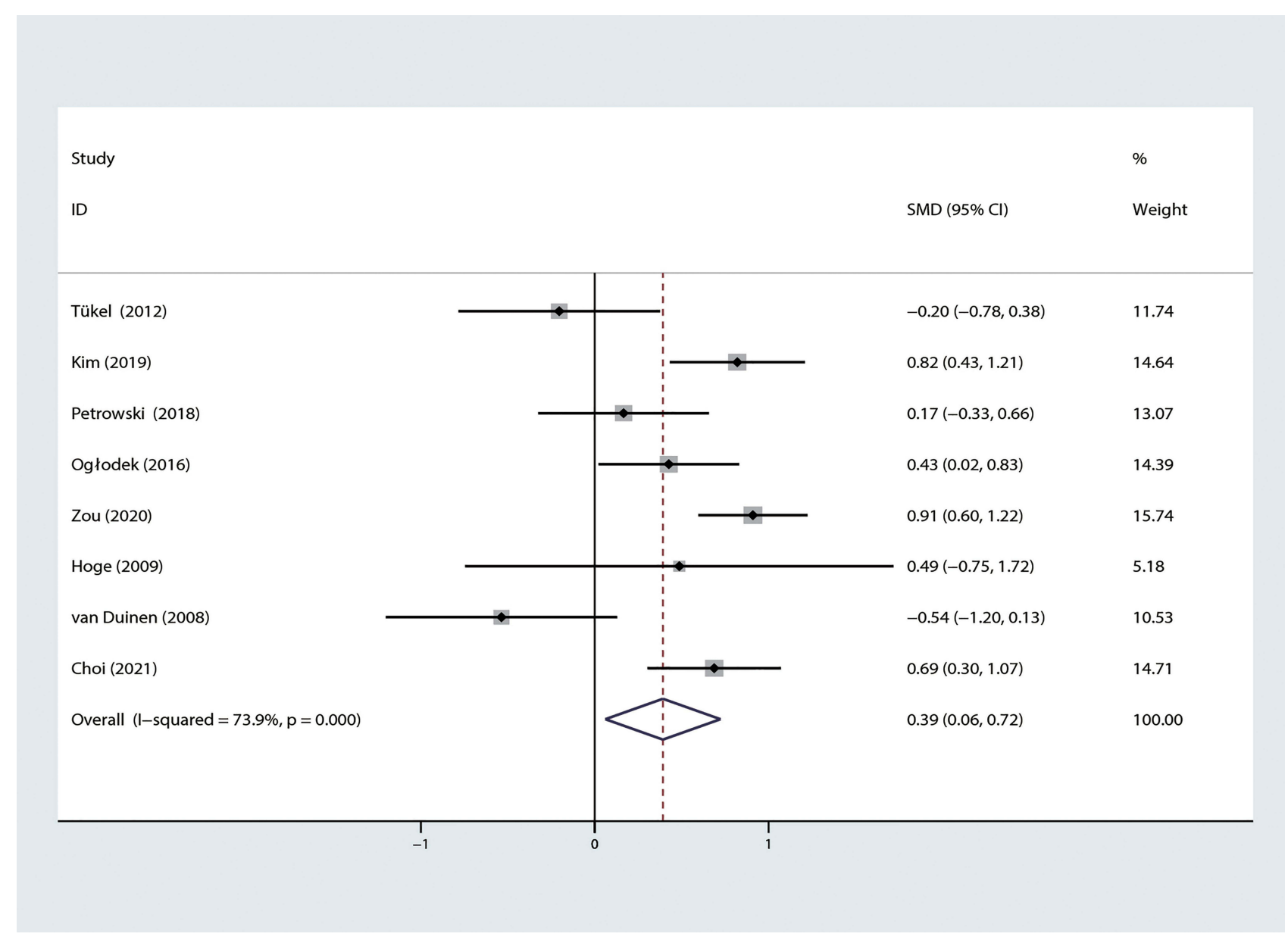

Figure 3 Forest plot of standard mean difference (SMD) of IL-6 levels in PD vs controls.

Abbreviations: IL-6, interleukin-6; PD, panic disorder.

compared IL-4 levels in PD patients and controls, and one demonstrated a higher level of IL-4 in PD patients, ${ }^{13}$ while the other did not identify a significant difference after adjusting for age, sex, BMI, educational level, resident location, marital status and smoking. ${ }^{22}$ IL-12 and IL-18 levels were observed between PD patients and controls only in one study, and it has been shown that IL-12 levels are lower ${ }^{23}$ and IL-18 levels are higher in PD patients. ${ }^{28}$ Another study compared the relative levels of IL-5, IL-7, IL-13 and IL-15 between PD patients and controls. The data showed that PD patients presented higher levels of IL-7 and IL-13 than controls, while no significant differences were obtained in the levels of IL-5 and IL-15. ${ }^{13}$

\section{TNF- $\alpha$}

Six studies involving 207 PD patients and 204 controls examined TNF- $\alpha$ levels. Only Hoge et $\mathrm{al}^{13}$ showed a significantly higher level of TNF- $\alpha$ in PD patients than in controls $(P<0.0005)$, while the remaining 5 studies all considered no significant difference between the two groups. $^{21-23,25,29}$ Our meta-analysis showed a higher level of TNF- $\alpha$ in PD patients $(\mathrm{z}=2.51, p=0.012)$, with a small effect size and less heterogeneity $\left(\mathrm{I}^{2}=0.0 \%, \mathrm{df}=\right.$ $5, p=0.876$ ) (Figure 7).

\section{INF- $\gamma$}

We retrieved 4 studies on the correlation between PD and IFN- $\gamma$, involving 176 PD patients and 175 controls. Tükel et $\mathrm{al}^{23}$ reported a significantly lower level of IFN- $\gamma$ in PD patients than in controls, and a reduced level of IFN- $\gamma$ was a significant predictive factor for PD $(P=0.04)$. Inconsistently, Zou et $\mathrm{al}^{22}$ did not identify a significant difference in IFN- $\gamma$ levels between groups. Hoge et $\mathrm{al}^{13}$ and Choi et $\mathrm{al}^{21}$ obtained similar results that detected no significant difference in baseline IFN- $\gamma$ between PD patients and controls. Through meta-analysis, our findings showed no significant difference in IFN- $\gamma$ levels between groups $(\mathrm{z}=0.10, p=0.921)$, and the heterogeneity among 


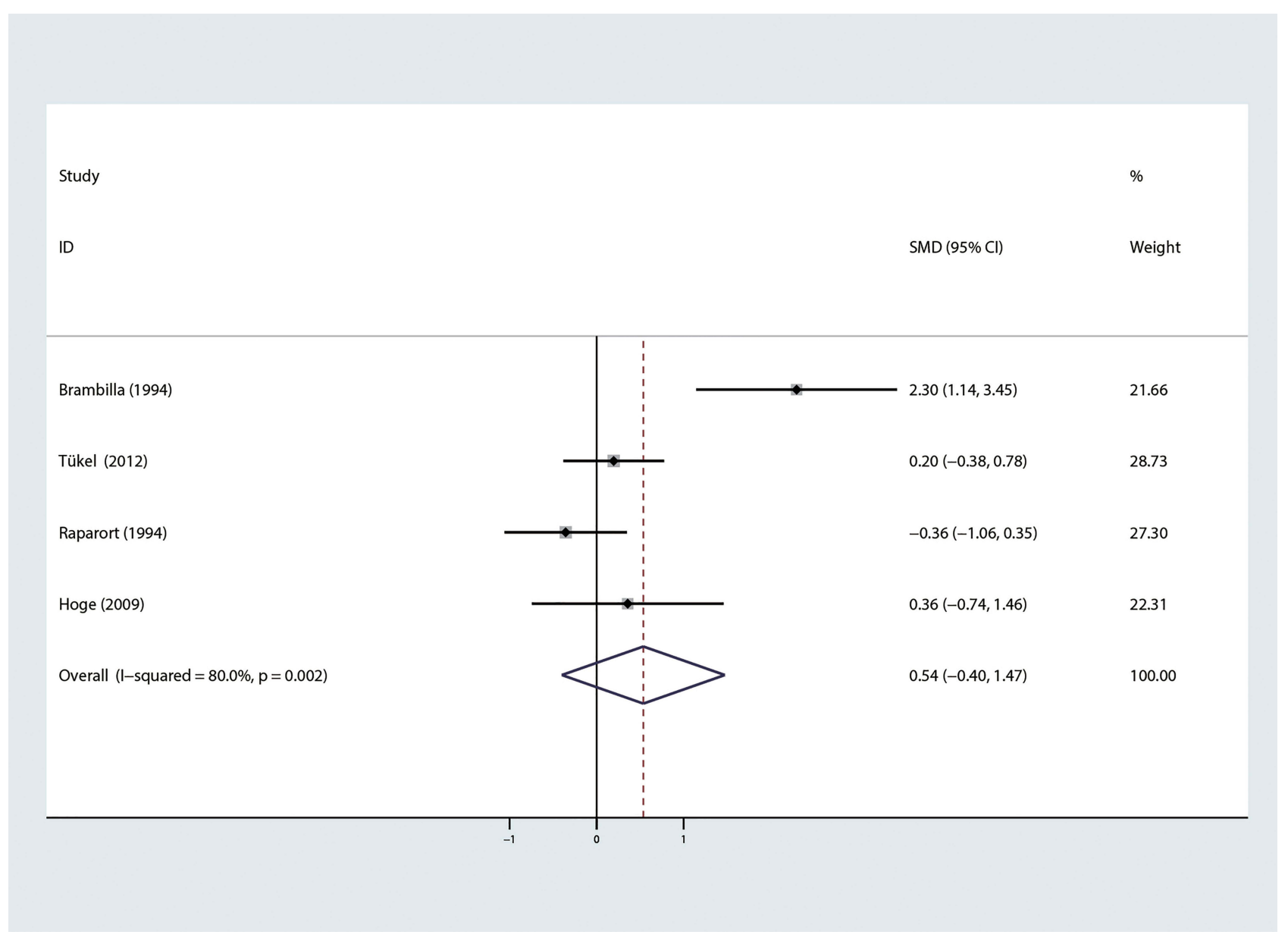

Figure 4 Forest plot of standard mean difference (SMD) of IL- I $\beta$ levels in PD vs controls.

Abbreviations: IL-I $\beta$, interleukin-I $\beta$; PD, panic disorder.

the 4 studies was relatively high $\left(\mathrm{I}^{2}=80.6 \%, \mathrm{df}=3, p=\right.$ 0.001) (Figure 8).

\section{Discussion}

Although the inflammatory process has been verified to be associated with the pathophysiology of PD, inflammatory marker levels in PD patients have not been well analyzed. ${ }^{12,30,31}$ To our knowledge, this was the first systematic review and meta-analysis focusing on peripheral inflammatory markers in PD patients. Through a literature review, a total of 14 eligible studies involving 18 inflammatory markers in 1455 PD patients were included. The overall results to date have shown a trend of elevated peripheral cytokine levels in PD patients, but the results have been very inconsistent. In the present study, a metaanalysis was conducted on inflammatory markers in PD patients reported in three or more studies. Our results demonstrated that peripheral levels of CRP, IL-6, IL-2, and TNF- $\alpha$ were significantly higher in PD patients than in healthy controls, while no significant differences in the levels of IL-1 $\beta$, IL-10 and IFN- $\gamma$ were detected.

$\mathrm{CRP}$ is a key early proinflammatory surveillance molecule involved in activation of the complement system and immune system. ${ }^{32}$ Anxiety-related psychological stress has been proven to activate the inflammatory response, which is related to increased levels of circulating inflammation markers such as $\mathrm{CRP}^{33,34}$ Our meta-analysis results showed higher CRP levels in PD patients than in controls ( $\mathrm{SMD}=0.41)$. The effect size of CRP was higher than that reported in other anxiety disorders, such as posttraumatic stress disorder (PTSD, $\mathrm{SMD}=-0.14)^{35}$ or major depressive disorder (MDD, $\mathrm{SMD}=0.14),{ }^{36}$ while it was similar to that of schizophrenia $(\mathrm{SMD}=0.45)^{37}$ and generalized anxiety disorder (GAD, $\mathrm{SMD}=0.38) .{ }^{16}$ Among the 4 studies reporting CRP levels in PD patients, only one study ${ }^{18}$ concluded that the CRP level in PD patients was higher than that in the control group, but a trend of elevated CRP levels in PD patients could be observed in 


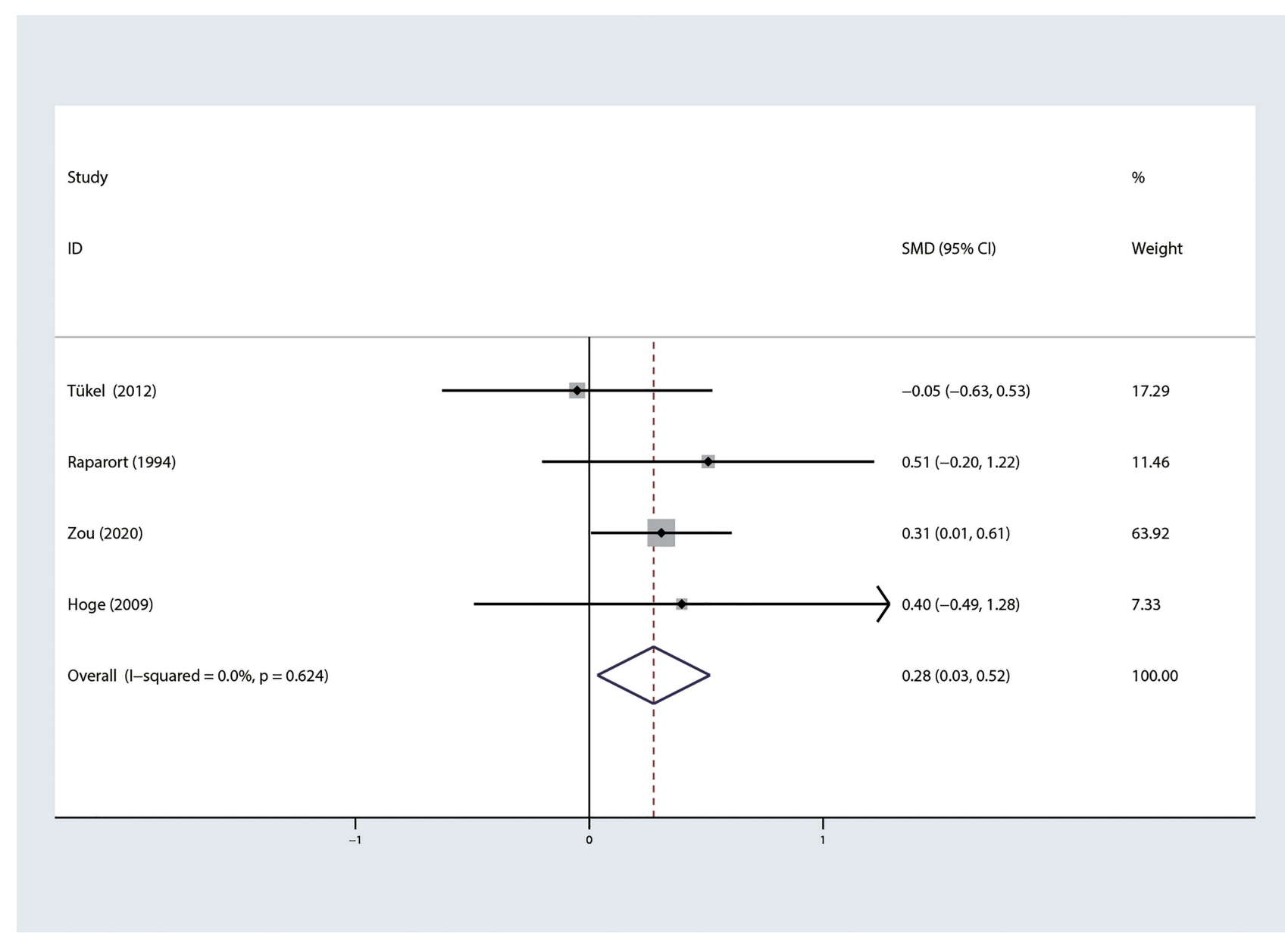

Figure 5 Forest plot of standard mean difference (SMD) of IL-2 levels in PD vs controls.

Abbreviations: IL-2, interleukin-2; PD, panic disorder.

the other three studies. ${ }^{19-21}$ Although this conclusion is based on the analysis of a small sample, our conclusion is consistent with the results of a large-scale study involving 1698 PD patients and 48,677 control subjects. ${ }^{38}$ However, this study was excluded from our study because some PD patients also had clinical diseases.

Every member of the IL family is of great significance for inflammation regulation. As an important proinflammatory cytokine, IL-6 is one of the most initially reported cytokines involved in the neuroimmune inflammatory response, ${ }^{24}$ which induces acute phase protein synthesis, regulation of proinflammatory factors and activation of microglia. ${ }^{39}$ The role of IL-6 in mental disorders and neuroinfectious diseases has been validated. ${ }^{13,40}$ Elevated IL-6 levels are associated with many mental disorders, including depression, schizophrenia, and PTSD. ${ }^{32}$ It has been reported that the severity of PD is positively correlated with IL-6 levels. ${ }^{19,41}$ Our meta-analysis found that PD patients had a higher level of IL- 6 than controls
( $\mathrm{SMD}=0.39$ ), and the heterogeneity of relevant studies was high. At present, immunotherapy for mental disorders is already under way. Serving as a therapeutic target for autoimmune diseases, pharmacological blockade of IL-6 is used to treat several autoimmune diseases, such as rheumatoid arthritis (RA). ${ }^{32}$ Therefore, it is reasonable to speculate that monitoring dynamic levels of IL-6 in PD patients is of significance to the diagnosis and treatment of PD.

IL-2 is secreted by activated T cells and plays an important role in $\mathrm{T}$ cell-mediated autoimmune diseases and inflammation. ${ }^{32}$ IL-10 is an anti-inflammatory cytokine secreted by $\mathrm{T}$ helper cells that contributes to regulating the immune response by limiting the proinflammatory process. ${ }^{42}$ Research results on IL-2 and IL-10 in PD patients are inconsistent. Although most studies have suggested that IL-2 and IL-10 are not correlated with PD, our meta-analysis, interestingly, revealed higher levels of IL-2 in PD patients than in controls, but the effect size was small $(\mathrm{SMD}=0.28)$. We 


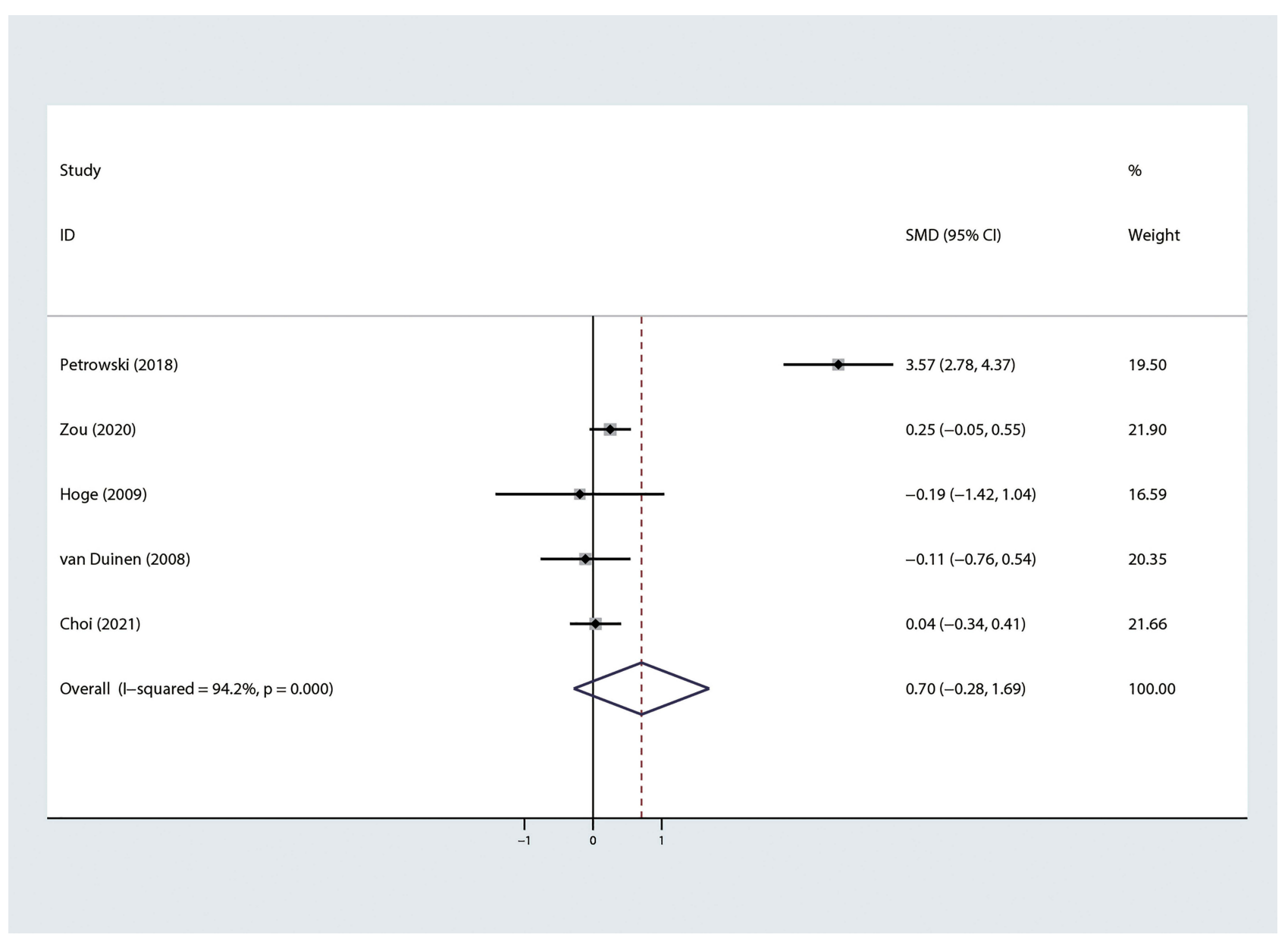

Figure 6 Forest plot of standard mean difference (SMD) of IL-10 levels in PD vs controls.

Abbreviations: IL-10, interleukin-I0; PD, panic disorder.

believe that our finding is reasonable because PD is closely related to the inflammatory response. ${ }^{13}$ However, the current epidemiological evidence on the correlation between PD and ILs is limited and should be further confirmed by prospective longitudinal studies.

TNF- $\alpha$ is also a proinflammatory cytokine with extensive functions in host defense. It can initiate a strong acute inflammatory response. ${ }^{43}$ As a blocking target of monoclonal antibodies, TNF- $\alpha$ can be used in the treatment of multiple autoimmune diseases, such as Crohn's disease and RA. ${ }^{32}$ It has been reported that anxiety disorders can activate the inflammatory response, and regulate the hypothalamicpituitary-adrenal (HPA) system by upregulating TNF- $\alpha .^{33}$ Brambilla et $\mathrm{al}^{29}$ believed that the high secretion of norepinephrine in PD patients can downregulate the density of norepinephrine receptors and weaken the inhibitory effect of the immune system, thus stimulating the production of cytokines such as TNF- $\alpha$. Our meta-analysis results also revealed that PD patients had a higher level of TNF- $\alpha$ than controls
( $\mathrm{SMD}=0.25$ ), and the heterogeneity of relevant studies was small. Among the 6 studies on TNF- $\alpha$, only one conducted by Hoge et $\mathrm{al}^{13}$ reported a higher level of TNF- $\alpha$ in PD patients than in controls, while the other 5 studies $^{21-23,25,29}$ all found that the value of TNF- $\alpha$ in PD patients was higher than that in the control group. This was similar to the meta-analysis of CRP, and more studies are needed to validate our results.

Although similar measurement methods were adopted in the included studies, the detection types were not all the same. We attempted to control confounding factors as much as possible, and as a result, the final sample size was small. The adjustment of important confounding factors differed among these studies, including comorbid anxiety or depression, drug use, sample collection and blood collection time, which can all cause high heterogeneity. A further limitation was that none of the included studies were labeled with cutoff values for inflammatory markers. This also increased the heterogeneity of the studies. Due to the lack of studies, we were unable to analyze the causes of heterogeneity. In addition, we found that there 


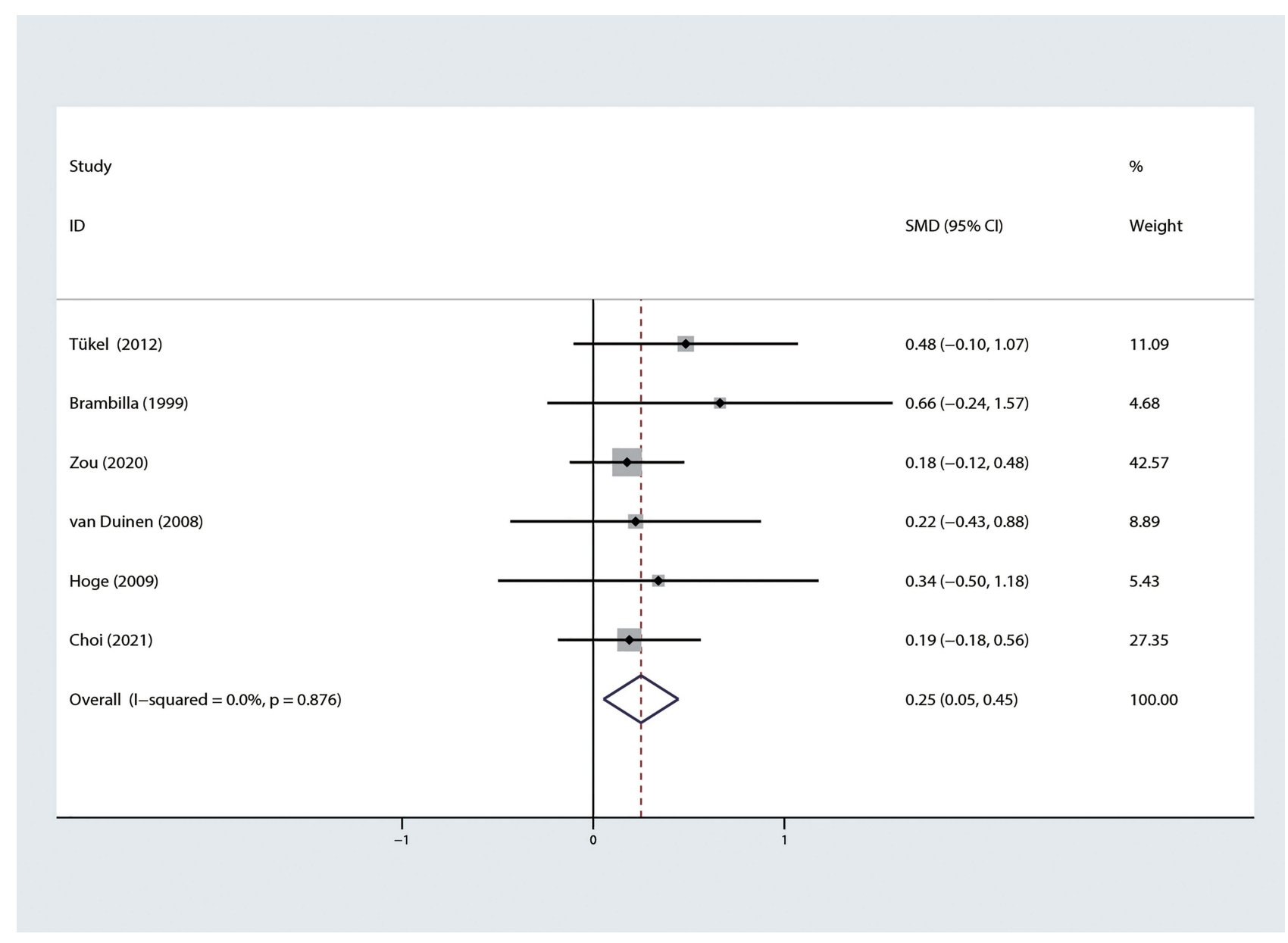

Figure 7 Forest plot of standard mean difference (SMD) of TNF- $\alpha$ levels in PD vs controls.

Abbreviations: TNF- $\alpha$, tumor necrosis factor- $\alpha$; PD, panic disorder.

are few studies on the correlation between the severity and duration of PD and inflammatory markers, which makes it impossible to analyze whether these inflammatory markers can predict prognosis. Finally, peripheral blood samples analyzed in this meta-analysis were limited as indicators of the inflammatory response in the brain, because the inflammatory response involving cytokines may be confined to the cerebrospinal fluid, rather than a broader systemic response. In conclusion, our findings should be cautiously interpreted and validated in further studies because of the high heterogeneity, small sample size, and different adjustments in known confounding factors of PD (eg, BMI, smoking, drug use, and comorbid anxiety or depression).

\section{Conclusions}

Although the role of inflammatory cytokines in the pathophysiology of PD has not yet been clearly clarified, preliminary evidence has shown an elevated inflammatory response in PD patients. Compared with other mental disorders, such as major depression and schizophrenia, neuroinflammation research on PD is scant. This meta-analysis obtained a preliminary conclusion that the inflammatory state was common in PD patients, manifested by an extensive increase in peripheral proinflammatory cytokines. However, it was unclear whether the inflammatory response in PD patients was the key pathological event of PD, or the stress response secondary to PD. Although we are still a long way from using inflammatory cytokines as biomarkers or therapeutic targets for PD, the current findings reflect the role that persistent inflammation plays in the development of PD. In the future, great efforts should be made to explore the potential of inflammatory cytokines as biomarkers or therapeutic targets of PD. In addition, large-scale prospective longitudinal studies or repeat studies in larger samples are needed to further validate the correlation between PD and inflammation and the underlying mechanism. 


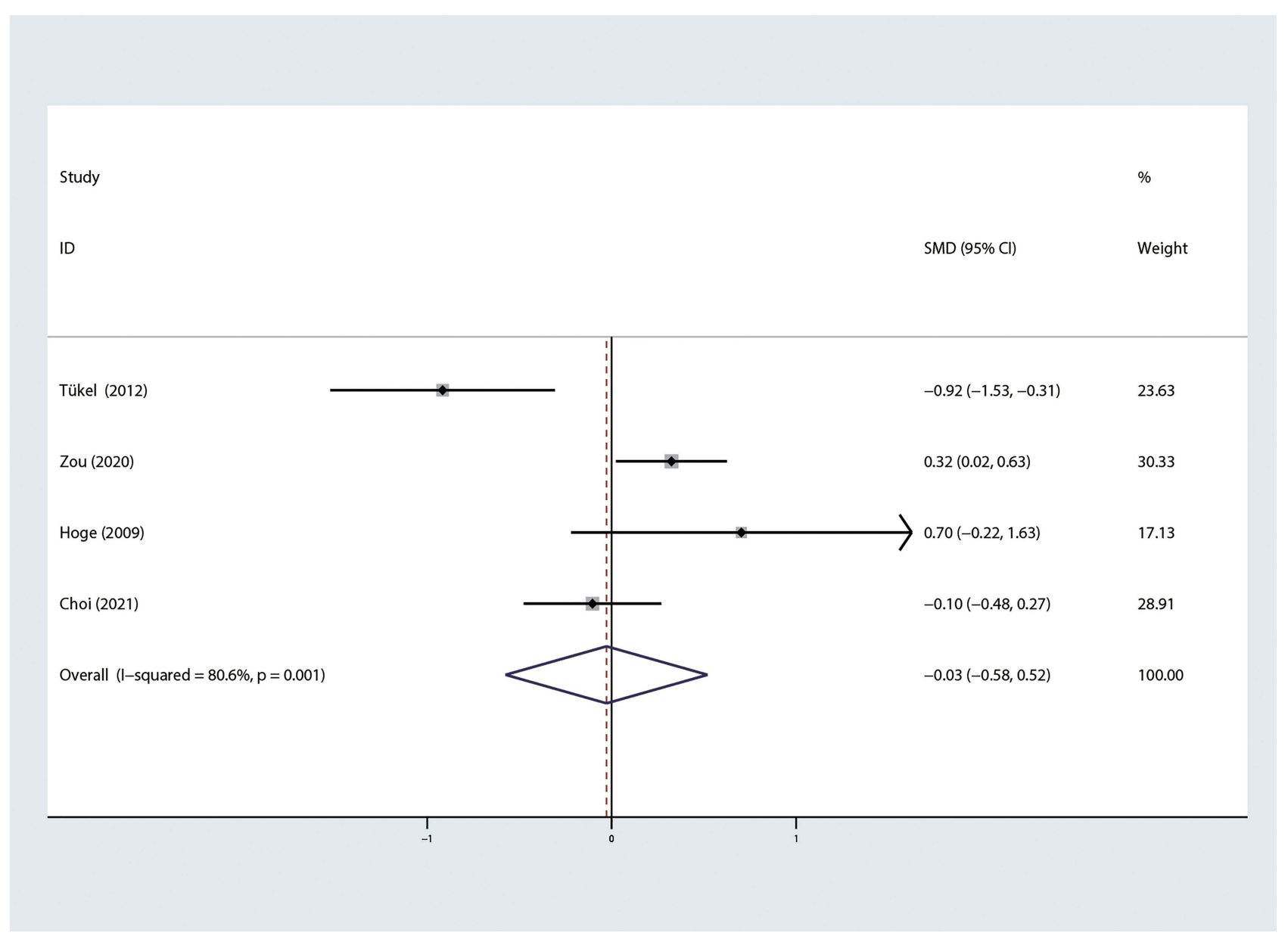

Figure 8 Forest plot of standard mean difference (SMD) of IFN- $\gamma$ levels in PD vs controls.

Abbreviations: IFN $-\gamma$, interferon- $\gamma$; PD, panic disorder.

\section{Abbreviations}

PD, panic disorder; PRISMA, preferred reporting items for systematic reviews and meta-analyses; BMI, body mass index; SMD, standardized mean difference; CI, confidence interval; ELISA, enzyme-linked immunosorbent assay; RA, rheumatoid arthritis; HPA, hypothalamic-pituitary-adrenal; CRP, C-reactive protein; IL, interleukin; TNF- $\alpha$, tumor necrosis factor- $\alpha$; IFN- $\gamma$, interferon- $\gamma$; PTSD, posttraumatic stress disorder; MDD, major depressive disorder.

\section{Data Sharing Statement}

All datasets used and/or analyzed during the current study are available from the corresponding author on reasonable request.

\section{Author Contributions}

All authors made a significant contribution to the work reported, whether that is in the conception, study design, execution, acquisition of data, analysis and interpretation, or in all these areas; took part in drafting, revising or critically reviewing the article; gave final approval of the version to be published; have agreed on the journal to which the article has been submitted; and agree to be accountable for all aspects of the work.

\section{Funding}

This research did not receive any funding support.

\section{Disclosure}

The authors report no conflicts of interest related to this work.

\section{References}

1. Réus GZ, Fries GR, Stertz L, et al. The role of inflammation and microglial activation in the pathophysiology of psychiatric disorders. Neuroscience. 2015;300:141-154. doi:10.1016/j.neuroscience.2015.05.018 
2. Khandaker GM, Pearson RM, Zammit S, Lewis G, Jones PB. Association of serum interleukin 6 and $\mathrm{C}$-reactive protein in childhood with depression and psychosis in young adult life: a population-based longitudinal study. JAMA Psychiatry. 2014;71(10):1121-1128. doi:10.1001/ jamapsychiatry.2014.1332

3. Dantzer R, O'Connor JC, Freund GG, Johnson RW, Kelley KW. From inflammation to sickness and depression: when the immune system subjugates the brain. Nat Rev Neurosci. 2008;9(1):46-56. doi:10.1038/nrn2297

4. Kessler RC, Berglund P, Demler O, Jin R, Merikangas KR, Walters EE. Lifetime prevalence and age-of-onset distributions of DSM-IV disorders in the National Comorbidity Survey Replication. Arch Gen Psychiatry. 2005;62(6):593-602. doi:10.1001/archpsyc.62. 6.593

5. Beesdo K, Pine DS, Lieb R, Wittchen HU. Incidence and risk patterns of anxiety and depressive disorders and categorization of generalized anxiety disorder. Arch Gen Psychiatry. 2010;67 (1):47-57.

6. Nepon J, Belik SL, Bolton J, Sareen J. The relationship between anxiety disorders and suicide attempts: findings from the National Epidemiologic Survey on Alcohol and Related Conditions. Depress Anxiety. 2010;27(9):791-798. doi:10.1002/da.20674

7. Batelaan NM, Rhebergen D, de Graaf R, Spijker J, Beekman AT, Penninx BW. Panic attacks as a dimension of psychopathology: evidence for associations with onset and course of mental disorders and level of functioning. J Clin Psychiatry. 2012;73(9):1195-1202. doi: $10.4088 / \mathrm{JCP} .12 \mathrm{~m} 07743$

8. Schiepers OJ, Wichers MC, Maes M. Cytokines and major depression. Prog Neuropsychopharmacol Biol Psychiatry. 2005;29 (2):201-217. doi:10.1016/j.pnpbp.2004.11.003

9. Michopoulos V, Powers A, Gillespie CF, Ressler KJ, Jovanovic T. Inflammation in fear- and anxiety-based disorders: PTSD, GAD, and beyond. Neuropsychopharmacology. 2017;42(1):254-270. doi:10.103 8/npp.2016.146

10. Luo Y, He H, Zhang M, Huang X, Fan N. Altered serum levels of TNF- $\alpha$, IL- 6 and IL-18 in manic, depressive, mixed state of bipolar disorder patients. Psychiatry Res. 2016;244:19-23. doi:10.1016/j. psychres.2016.07.027

11. Luo Y, He H, Zhang J, Ou Y, Fan N. Changes in serum TNF- $\alpha$, IL18, and IL-6 concentrations in patients with chronic schizophrenia at admission and at discharge. Compr Psychiatry. 2019;90:82-87. doi:10.1016/j.comppsych.2019.01.003

12. Quagliato LA, Nardi AE. Cytokine alterations in panic disorder: a systematic review. J Affect Disord. 2018;228:91-96. doi:10.1016/ j.jad.2017.11.094

13. Hoge EA, Brandstetter K, Moshier S, Pollack MH, Wong KK, Simon NM. Broad spectrum of cytokine abnormalities in panic disorder and posttraumatic stress disorder. Depress Anxiety. 2009;26 (5):447-455. doi:10.1002/da.20564

14. Salim S, Chugh G, Asghar M. Inflammation in anxiety. Adv Protein Chem Struct Biol. 2012;88:1-25.

15. Petrowski K, Wichmann S, Kirschbaum C. Stress-induced pro- and anti-inflammatory cytokine concentrations in panic disorder patients. Psychoneuroendocrinology. 2018;94:31-37. doi:10.1016/j.psyneuen. 2018.05.005

16. Costello H, Gould RL, Abrol E, Howard R. Systematic review and meta-analysis of the association between peripheral inflammatory cytokines and generalised anxiety disorder. BMJ Open. 2019;9(7): e027925. doi:10.1136/bmjopen-2018-027925

17. Higgins JP, Thompson SG, Deeks JJ, Altman DG. Measuring inconsistency in meta-analyses. BMJ. 2003;327(7414):557-560. doi:10.11 36/bmj.327.7414.557

18. Herrán A, Sierra-Biddle D, García-Unzueta MT, Puente J, VázquezBarquero JL, Antonio Amado J. The acute phase response in panic disorder. Int J Neuropsychopharmacol. 2005;8(4):529-535. doi:10. 1017/S1461145705005432
19. Kim K, Jang EH, Kim AY, et al. Pre-treatment peripheral biomarkers associated with treatment response in panic symptoms in patients with major depressive disorder and panic disorder: a 12-week follow-up study. Compr Psychiatry. 2019;95:152140. doi:10.1016/j. comppsych.2019.152140

20. Strawn JR, Vollmer LL, Kmj M, et al. Acid-sensing T cell death associated gene-8 receptor expression in panic disorder. Brain Behav Immun. 2018;67:36-41. doi:10.1016/j.bbi.2017.07.014

21. Choi KW, Jang EH, Kim AY, et al. Predictive inflammatory biomarkers for change in suicidal ideation in major depressive disorder and panic disorder: a 12-week follow-up study. J Psychiatr Res. 2021;133:73-81. doi:10.1016/j.jpsychires.2020.12.011

22. Zou Z, Zhou B, Huang Y, Wang J, Min W, Li T. Differences in cytokines between patients with generalised anxiety disorder and panic disorder. J Psychosom Res. 2020;133:109975. doi:10.1016/j. jpsychores.2020.109975

23. Tükel R, Arslan BA, Ertekin BA, et al. Decreased IFN- $\gamma$ and IL-12 levels in panic disorder. $J$ Psychosom Res. 2012;73(1):63-67. doi:10.1016/j.jpsychores.2012.04.012

24. Ogłodek EA, Szota AM, Just MJ, Szromek AR, Araszkiewicz A. A study of chemokines, chemokine receptors and interleukin-6 in patients with panic disorder, personality disorders and their co-morbidity. Pharmacol Rep. 2016;68(4):756-763. doi:10.1016/j. pharep.2016.03.017

25. van Duinen MA, Schruers KR, Kenis GR, et al. Effects of experimental panic on neuroimmunological functioning. $J$ Psychosom Res. 2008;64(3):305-310. doi:10.1016/j.jpsychores.2007.10.004

26. Brambilla F, Bellodi L, Perna G, Bertani A, Panerai A, Sacerdote P. Plasma interleukin-1 beta concentrations in panic disorder. Psychiatry Res. 1994;54(2):135-142. doi:10.1016/0165-1781(94)90002-7

27. Rapaport MH, Stein MB. Serum cytokine and soluble interleukin-2 receptors in patients with panic disorder. Anxiety. 1994;1(1):22-25. doi:10.1002/anxi.3070010106

28. Kokai M, Kashiwamura S, Okamura H, Ohara K, Morita Y. Plasma interleukin-18 levels in patients with psychiatric disorders. J Immunother. 2002;25(Suppl 1):S68-71. doi:10.1097/00002371200203001-00011

29. Brambilla F, Bellodi L, Perna G. Plasma levels of tumor necrosis factor-alpha in patients with panic disorder: effect of alprazolam therapy. Psychiatry Res. 1999;89(1):21-27. doi:10.1016/S0165-1781(99)00091-8

30. de la Fontaine L, Schwarz MJ, Eser D, Müller N, Rupprecht R, Zwanzger P. Effects of experimentally induced panic attacks on neuroimmunological markers. J Neural Transm. 2009;116(6):69 9-702. doi:10.1007/s00702-008-0140-6

31. Glaus J, von Känel R, Lasserre AM, et al. The bidirectional relationship between anxiety disorders and circulating levels of inflammatory markers: results from a large longitudinal population-based study. Depress Anxiety. 2018;35(4):360-371. doi:10.1002/da.22710

32. Akdis M, Aab A, Altunbulakli C, et al. Interleukins (from IL-1 to IL38 ), interferons, transforming growth factor $\beta$, and TNF- $\alpha$ : receptors, functions, and roles in diseases. J Allergy Clin Immunol. 2016;138 (4):984-1010. doi:10.1016/j.jaci.2016.06.033

33. Furtado M, Katzman MA. Neuroinflammatory pathways in anxiety, posttraumatic stress, and obsessive compulsive disorders. Psychiatry Res. 2015;229(1-2):37-48. doi:10.1016/j.psychres.2015.05.036

34. Steptoe A, Hamer M, Chida Y. The effects of acute psychological stress on circulating inflammatory factors in humans: a review and meta-analysis. Brain Behav Immun. 2007;21(7):901-912. doi:10.10 16/j.bbi.2007.03.011

35. Passos IC, Vasconcelos-Moreno MP, Costa LG, et al. Inflammatory markers in post-traumatic stress disorder: a systematic review, meta-analysis, and meta-regression. Lancet Psychiatry. 2015;2 (11):1002-1012. doi:10.1016/S2215-0366(15)00309-0

36. Howren MB, Lamkin DM, Suls J. Associations of depression with C-reactive protein, IL-1, and IL-6: a meta-analysis. Psychosom Med. 2009;71(2):171-186. doi:10.1097/PSY.0b013e3181907c1b 
37. Miller BJ, Culpepper N, Rapaport MH. C-reactive protein levels in schizophrenia: a review and meta-analysis. Clin Schizophr Relat Psychoses. 2014;7(4):223-230. doi:10.3371/CSRP.MICU.020813

38. Naudé PJW, Roest AM, Stein DJ, de Jonge P, Doornbos B. Anxiety disorders and CRP in a population cohort study with 54,326 participants: the LifeLines study. World J Biol Psychiatry. 2018;19 (6):461-470. doi:10.1080/15622975.2018.1433325

39. Audet MC, Anisman H. Interplay between pro-inflammatory cytokines and growth factors in depressive illnesses. Front Cell Neurosci. 2013;7:68.

40. Guyon A, Massa F, Rovère C, Nahon JL. How cytokines can influence the brain: a role for chemokines? J Neuroimmunol. 2008;198(12):46-55. doi:10.1016/j.jneuroim.2008.04.009
41. Belem da Silva CT, Costa MA, Bortoluzzi A, et al. Cytokine levels in panic disorder: evidence for a dose-response relationship. Psychosom Med. 2017;79(2):126-132. doi:10.1097/ PSY.0000000000000384

42. Hofmann SR, Rösen-Wolff A, Tsokos GC, Hedrich CM. Biological properties and regulation of IL-10 related cytokines and their contribution to autoimmune disease and tissue injury. Clin Immunol. 2012;143(2):116-127. doi:10.1016/j.clim.2012.02. 005

43. Kataranovski M, Magić Z, Pejnović N. Early inflammatory cytokine and acute phase protein response under the stress of thermal injury in rats. Physiol Res. 1999;48(6):473-482.

\section{Publish your work in this journal}

Neuropsychiatric Disease and Treatment is an international, peerreviewed journal of clinical therapeutics and pharmacology focusing on concise rapid reporting of clinical or pre-clinical studies on a range of neuropsychiatric and neurological disorders. This journal is indexed on PubMed Central, the 'PsycINFO' database and CAS, and is the official journal of The International Neuropsychiatric Association (INA). The manuscript management system is completely online and includes a very quick and fair peer-review system, which is all easy to use. Visit http://www.dovepress.com/testimonials.php to read real quotes from published authors. 